\title{
Modelling Compressible Blood Flow with Slip in A Constricted Rectangular Flow Domain
}

\author{
by \\ Matthew DeClerico \\ Bachelor of Science, McMaster University, 2008
}

\author{
A thesis \\ presented to Ryerson University \\ in partial fulfillment of the \\ requirements for the degree of \\ Master of Science \\ in the Program of \\ Applied Mathematics
}

Toronto, Ontario, Canada, 2015

(C)Matthew DeClerico 2015 


\section{AUTHOR'S DECLARATION FOR ELECTRONIC SUBMISSION OF A THESIS}

I hereby declare that I am the sole author of this thesis. This is a true copy of the thesis, including any required final revisions, as accepted by my examiners.

I authorize Ryerson University to lend this thesis to other institutions or individuals for the purpose of scholarly research.

I further authorize Ryerson University to reproduce this thesis by photocopying or by other

means, in total or in part, at the request of other institutions or individuals for the purpose of scholarly research.

I understand that my thesis may be made electronically available to the public. 


\begin{abstract}
Master of Science 2015

Matthew DeClerico

Applied Mathematics

Ryerson University
\end{abstract}

Modelling Compressible Blood Flow with Slip in a Constricted Rectangular Flow Domain

The abnormal narrowing of blood vessels is known to affect the characterization of blood flow through these constricted regions. Both theoretical and clinical research has suggested that these changes in flow are associated with cardiovascular related diseases. Analytic, numerical, and particle based methods have been employed to solve the Navier-Stokes momentum integral equations associated with compressible, Newtonian fluid flow. In this thesis, the Karman-Pohlhausen method is used to transform a system of partial differential equations into a single second-order, non-linear differential equation in terms of the density. Numerical solutions are presented and important flow features, including the role of slip and compressibility, are discussed. The choice to use a symmetric rectangular channel, rather than a cylindrical one, is largely motivated by the opportunity to compare the numerical solutions with experimental data collected from a rectangular microchannel. The numerical results also indicate similar trends in the flow characteristics for the rectangular channel as compared to previous results using cylindrical models. 


\section{Acknowledgements}

First and foremost, I want to thank my supervisor Dr. Katrin Rohlf for her patience, guidance, and motivation. Her continuous support was invaluable through the entire research and writing process. More importantly, she helped me develop a confidence in myself that I did not have before. For that, I am extremely grateful. I also want to thank my supervisory committee for volunteering their time. A special thank you to Dr. Katrin Rohlf, Dr. Silvana Ilie, Dr. Jean-Paul Pascal, and all my course teachers for providing me with a strong foundation to be successful in my coursework, research, and future career opportunities.

I am thankful for the department of Mathematics at Ryerson. The faculty and staff were very helpful and always willing to provide me with both academic and professional support. I am also very thankful to my classmates and colleagues. I enjoyed working with them and appreciate the support they gave me to be successful in my classes and research. Each of them contributed in making my experience at Ryerson a positive and valuable one. 


\section{Contents}

1 Introduction $\quad 1$

2 Fluid Dynamics $\quad 4$

2.1 Governing Equations . . . . . . . . . . . . . . . . . . 5

2.2 Navier-Stokes in Cartesian Coordinates _ . . . . . . . . . . . . . . . 6

2.3 Flow Geometry . . . . . . . . . . . . . . . . . . . 9

3 Analytic Methods $\quad 12$

3.1 The Assumed Solution for Velocity . . . . . . . . . . . . . . . . . 12

3.2 Flow Rate Analysis . . . . . . . . . . . . . . . . . . . . . . . . . . 14

3.3 The Density Gradient . . . . . . . . . . . . . . . . . . . . 15

3.4 Upstream Analysis . . . . . . . . . . . . . . . . . . . . . . . 19

4 Numerical Solutions $\quad 21$

4.1 Role of Slip Velocity . . . . . . . . . . . . . . . . . . . . . . 22

4.2 Role of Compressibility . . . . . . . . . . . . . . . . . . . 22

4.3 Role of Geometry . . . . . . . . . . . . . . . . . . . . . . . 23

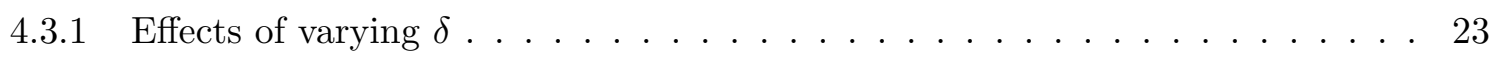

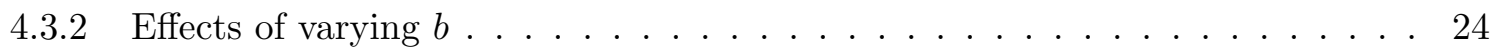

4.4 Role of Reynolds Number . . . . . . . . . . . . . . . . . . . . . . . . 24

4.5 Role of second-order derivative . . . . . . . . . . . . . . . . . 25

4.6 Graphical Results . . . . . . . . . . . . . . . . . . . . . . . 26

5 Conclusions and Future Work $\quad 34$

$\begin{array}{ll}\text { References } & 48\end{array}$ 


\section{List of Figures}

2.1 Flow geometry modelled as a rectangular microchannel. . . . . . . . . . . . . 9

2.2 Cross-section of flow domain using Gaussian distribution profile for the boundary. . 11

4.1 Solution for scaled density while varying slip velocity, $v_{s_{0}}$, with $M a_{0}=0.1$ and

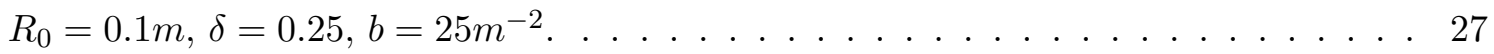

4.2 Solution for density gradient while varying slip velocity, $v_{s_{0}}$, with $M a_{0}=0.1$ and $R_{0}=0.1 m, \delta=0.25, b=25 m^{-2} \ldots \ldots \ldots \ldots \ldots \ldots \ldots \ldots \ldots$

4.3 Solution for scaled density while varying Mach Number, $M a$, with $v_{s_{0}}=0.1$ and $R_{0}=0.1 m, \delta=0.25, b=25 m^{-2}$

4.4 Solution for density gradient while varying Mach Number, $M a$, with $v_{s_{0}}=0.1$ and $R_{0}=0.1 m, \delta=0.25, b=25 m^{-2} \ldots \ldots \ldots \ldots \ldots \ldots \ldots$

4.5 Solution for scaled density while varying Mach Number, $M a$, with $v_{s_{0}}=0$ and $R_{0}=0.1 m, \delta=0.25, b=25 m^{-2}$.

4.6 Solution for density gradient while varying Mach Number, $M a$, with $v_{s_{0}}=0$ and $R_{0}=0.1 m, \delta=0.25, b=25 m^{-2}$

4.7 Solution for scaled density while varying severity of constriction, $\delta$, with with $M a_{0}=$ $0.1, v_{s_{0}}=0.1, R e=270$ and $R_{0}=0.1 \mathrm{~m}, b=25 \mathrm{~m}^{-2}$.

4.8 Solution for density gradient while varying varying severity of constriction, $\delta$, with $M a_{0}=0.1, v_{s_{0}}=0.1, R e=270$ and $R_{0}=0.1 \mathrm{~m}, b=25 \mathrm{~m}^{-2}$.

4.9 Solution for scaled density while varying width of constriction, $b$, with $M a_{0}=0.1$, $v_{s_{0}}=0.1, R e=270$ and $R_{0}=0.1 \mathrm{~m}, \delta=0.25 \mathrm{~m}^{-2}$.

4.10 Solution for density gradient while varying width of constriction, $b$, with $M a_{0}=0.1$, $v_{s_{0}}=0.1, R e=270$ and $R_{0}=0.1 \mathrm{~m}, \delta=0.25 \mathrm{~m}^{-2}$.

4.11 Solutions for scaled density while varying Reynolds number, $R e$, with $M a_{0}=0.1$, $v_{s_{0}}=0.1$, and $R_{0}=0.1 m, \delta=0.25, b=25 m^{-2} \ldots \ldots \ldots \ldots \ldots$

4.12 Solutions for density gradient while varying Reynolds number, $R e$, with $M a_{0}=0.1$, $v_{s_{0}}=0.1$, and $R_{0}=0.1 m, \delta=0.25, b=25 m^{-2} \ldots \ldots \ldots \ldots \ldots \ldots \ldots$ 
4.13 Solutions for scaled density for first order DE compared to second order DE with $M a_{0}=0.1, v_{s_{0}}=0, R e=300$ and $R_{0}=0.1 m, \delta=0.25, b=25 m^{-2} \ldots \ldots . \ldots$

4.14 Solutions for scaled density for first order DE compared to second order DE with $M a_{0}=0.1, v_{s_{0}}=0.1, R e=270$ and $R_{0}=0.1 m, \delta=0.25, b=25 m^{-2} \ldots \ldots 33$ 


\section{List of Appendices}

Appendix A - Additional Calculations $\quad 36$

A.1 Derivatives of Integrals . . . . . . . . . . . . . . . 36

A.2 Centerline Velocity . . . . . . . . . . . . . . . 37

A.3 Derivative of $V \ldots \ldots \ldots \ldots \ldots \ldots \ldots \ldots \ldots \ldots \ldots \ldots \ldots \ldots \ldots$

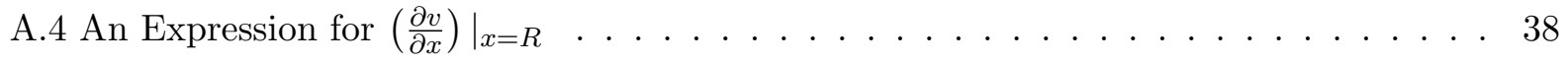

A.5 Reynolds Relationship . . . . . . . . . . . . . . . . . . 39

A.6 Density ODE . . . . . . . . . . . . . . . . . . . . . . . . . . 42 


\section{Chapter 1}

\section{Introduction}

A constriction in the artery, or arterial stenosis, is the abnormal narrowing of a blood vessel. When blood contains high levels of cholesterol and fatty molecules, they can adhere to the artery walls forming a plaque. Over time, these deposits grow narrowing the cross-sectional area of the artery. Alternatively, the stenosis can form as a result of arterial lesions along the vessel walls. Such constrictions alter the mechanical forces and flow properties of the blood, producing physiological, biochemical, and pathological changes in the body [24]. It is believed that the locations of these constrictions are not random; rather they are observed in neighbourhoods where the flow properties undergo large changes, such as areas of increased curvature or branching ([4], [15]). Multiple researchers have linked the changes in flow properties to cardiovascular related diseases. Improved understanding of the fluid dynamics of blood will allow better identification, treatment, and prevention of cardiovascular related diseases [3].

The fluid dynamics of blood have been investigated analytically using idealized, smooth cylindrical geometries. The momentum integral equations have been solved using perturbation methods ([21], [19]) and the Karman-Pohlhausen method ([9], [24], [23], [1]). In the latter, the solution to the velocity profile is assumed to be of a polynomial form. While [23] assumes a zero slip boundary condition, it was suggested by [16] to include slip velocity along the vessel wall. The role of slip was further investigated by ([14], [1], [20]). In [1], the role of slip was investigated with respect to 
an asymmetric constriction. In the Thesis by [20], the role of slip was investigated using a variety of smooth geometries.

Numerical methods have also been successfully implemented to better understand how fluid properties are affected by the stenosis ([13], [7]). It was numerically shown by [12] that at low Reynolds numbers, recirculation zones did not form downstream of the constriction. Furthermore, at low Reynolds numbers, [2] numerically showed that the flow resistance went unaltered when the stenosis was modelled as an irregular, non-smooth surface. This is a significant result since it is known that flows in stenotic neighbourhoods involve low Reynolds numbers [5], and the stenosis is often asymmetrical in shape [7].

Blood is often modelled as an incompressible, Newtonian fluid ([2], [22]). However, [3] and [19] demonstrated that the pressure gradient is affected by the weakly compressible nature of blood. Recently, particle-based methods called Multi-particle Collision Dynamics (MPC) have become popular approaches to further study the fluid properties of blood $([11],[3],[1])$. MPC methods allows researchers to describe the individual interactions among particles suspended in the blood. This approach is also favourable since compressibility is built into this model. The rise in popularity of particle-based methods is attributed to advancements in high performance computing [8]. Previously, such methods were unfavourable due to the immense processing resources needed to model individual particles in the fluid.

Early experimental research was carried out by [10] using a long, rigid tube with a theoretically modelled stenosis. In [6], the flow properties were compared using a straight, smooth tube and a cast of a diseased human artery. Later experiments ([17], [4]) investigated the properties of nonNewtonian fluids as they moved through a sudden constriction. The amount of experimental data to compare with the results of analytic, numerical, and particle based methods is growing, yet, it is still limited. This Thesis is largely motivated by the opportunity to compare numerical results with experimental data. A team of engineers plans to construct a rectangular microchannel to further study the dynamics of blood-like fluids moving through a constriction. For this reason a rectangular vessel is chosen to model the constriction instead of the more popular, and biologically 
relevant, cylindrical geometry.

In Chapter 2 the governing equations will be provided in Cartesian coordinates and initial assumptions, along with the full details of the model, will be discussed. Chapter 3 provides the specifics of the analytic methods used to develop a differential equation in terms of density. In Chapter 4, results are presented graphically along with discussion points. Finally, in Chapter 5, conclusions and possibilities for future projects are presented. 


\section{Chapter 2}

\section{Fluid Dynamics}

Fluid dynamics is the study of the movement, often referred to as flow, of fluids. A fluid is a substance that deforms continuously under external forces. There are two types of fluids; one being a gas, the other a liquid. Both gases and liquids are comprised of molecules and can flow easily, however they exhibit some defining differences. A gas has no fixed volume and will take the shape of its container. Gases are easily compressible since the molecules are widely spread out. In comparison, due to stronger attractive forces, the molecules in a liquid are more closely packed together. Liquids will take the shape of the container, but unlike gases, they have a definite volume. Fluid dynamics is an evolving and multidisciplinary subject. This subject is studied across many fields including biology, astronomy, engineering, and meteorology. Fluid properties, such as velocity, density, and pressure, are described by the solutions to the governing equations that arise from the laws of fluid flow.

In this Chapter, the governing equations describing compressible, Newtonian fluids are presented in Cartesian Coordinates. Initial assumptions that have been used in the literature are adopted to transform a system of partial differential equations into a non-linear, second order differential equation. Finally, a full description of the flow domain is discussed. 


\subsection{Governing Equations}

The goal is to derive a system of governing equations which describe the changes in physical properties (velocity, density, and pressure) of a fluid during flow. There are two approaches that have been used to achieve this. The first approach considers the individual molecules in the fluid. The macroscopic behaviour of the fluid is described by the dynamics of the molecules within. In a typical fluid system, the number of molecules is of the order $\sim 10^{9}$. Keeping track of how each molecule changes is very complex and computationally expensive. Instead, the second approach, called the continuum method, is used. In this model the fluid is assumed to be made up of homogeneous fluid particles that vary continuously in space and time. Each fluid particle is thought to be an average of the state of the system in a local neighbourhood. This approach allows for the interactions and changes of individual molecules in the system to be ignored.

The state of the system is described by the velocity vector $\mathbf{u}(\mathbf{x}, t)$, the density $\rho(\mathbf{x}, t)$, and the pressure $P(\mathbf{x}, t)$, where $\mathbf{x}=(x, y, z)$ are spatial coordinates and $t$ is time. For reference, bold face will be used to represent vector quantities. In addition to the continuum approach, all fluids are subject to conservative laws, namely conservation of momentum and conservation of mass. For an isothermal system, these laws lead us to a system of governing partial differential equations in terms of velocity, density, and pressure.

$$
\begin{aligned}
& \rho\left(\frac{\partial \mathbf{u}}{\partial t}+\mathbf{u} \cdot \nabla \mathbf{u}\right)=-\nabla P+\nabla \cdot \boldsymbol{\tau}+\mathbf{F} \\
& \boldsymbol{\tau}=\mu\left(\nabla \mathbf{u}+\nabla \mathbf{u}^{T}-\frac{2}{3}(\nabla \cdot \mathbf{u}) \mathbf{I}\right) \\
& \frac{\partial \rho}{\partial t}+\nabla \cdot(\rho \mathbf{u})=0 \\
& P=\frac{k_{B} T}{m} \rho
\end{aligned}
$$

Here $\nabla=\left(\frac{\partial}{\partial x}, \frac{\partial}{\partial y}, \frac{\partial}{\partial z}\right)$ is the gradient operator, $\mathbf{F}$ is the external force vector per unit volume acting on the fluid, $\mu$ is the dynamic viscosity, $T$ is the constant fluid temperature, $m$ is the mass 
of a fluid particle, and $k_{B}$ is the Boltzmann constant. Together, equation (2.1) and equation (2.2) are known as the Navier-Stokes equation for the conservation of momentum for isothermal, compressible, viscous fluid flow. The conservation of mass relationship takes the form of equation (2.3). Equation (2.4) is known as the Equation of State for an ideal gas and offers a means to translate between pressure and density in the system. Equations (2.1)-(2.4) represent a system of partial differential equations with unknowns $\mathbf{u}(\mathbf{x}, t), \rho(\mathbf{x}, t)$ and $P(\mathbf{x}, t)$.

\subsection{Navier-Stokes in Cartesian Coordinates}

For the model being used in this Thesis, the system of equations will need to be written in Cartesian coordinates. Following [18], the Navier-Stokes equation, (2.1) and (2.2), in component form for a fluid whose velocity is $\mathbf{u}=(u(x, y, z, t), v(x, y, z, t), w(x, y, z, t))$ in terms of the stress tensor, $\boldsymbol{\tau}$, can be written as:

$$
\begin{aligned}
& \rho\left(\frac{\partial u}{\partial t}+u \frac{\partial u}{\partial x}+v \frac{\partial u}{\partial y}+w \frac{\partial u}{\partial z}\right)=\left(\frac{\partial}{\partial x} \tau_{x x}+\frac{\partial}{\partial y} \tau_{y x}+\frac{\partial}{\partial z} \tau_{z x}\right)-\frac{\partial P}{\partial x}+\rho g_{x} \\
& \rho\left(\frac{\partial v}{\partial t}+u \frac{\partial v}{\partial x}+v \frac{\partial v}{\partial y}+w \frac{\partial v}{\partial z}\right)=\left(\frac{\partial}{\partial x} \tau_{x y}+\frac{\partial}{\partial y} \tau_{y y}+\frac{\partial}{\partial z} \tau_{z y}\right)-\frac{\partial P}{\partial y}+\rho g_{y} \\
& \rho\left(\frac{\partial w}{\partial t}+u \frac{\partial w}{\partial x}+v \frac{\partial w}{\partial y}+w \frac{\partial w}{\partial z}\right)=\left(\frac{\partial}{\partial x} \tau_{x z}+\frac{\partial}{\partial y} \tau_{y z}+\frac{\partial}{\partial z} \tau_{z z}\right)-\frac{\partial P}{\partial z}+\rho g_{z}
\end{aligned}
$$

Here, $\mathbf{F}=\rho\left(g_{x}, g_{y}, g_{z}\right)$. More specifically, $g_{x}$ denotes the component of the force in the $x$-direction. Continuing with [18], the components of the stress tensor are: 


$$
\begin{aligned}
& \tau_{x x}=\mu\left[2 \frac{\partial u}{\partial x}-\frac{2}{3}(\nabla \cdot \mathbf{u})\right], \\
& \tau_{y y}=\mu\left[2 \frac{\partial v}{\partial y}-\frac{2}{3}(\nabla \cdot \mathbf{u})\right], \\
& \tau_{z z}=\mu\left[2 \frac{\partial w}{\partial z}-\frac{2}{3}(\nabla \cdot \mathbf{u})\right], \\
& \tau_{x y}=\tau_{y x}=\mu\left(\frac{\partial u}{\partial y}+\frac{\partial v}{\partial x}\right), \\
& \tau_{y z}=\tau_{z y}=\mu\left(\frac{\partial v}{\partial z}+\frac{\partial w}{\partial y}\right), \\
& \tau_{x z}=\tau_{z x}=\mu\left(\frac{\partial u}{\partial z}+\frac{\partial w}{\partial x}\right), \\
& \nabla \cdot \mathbf{u}=\frac{\partial u}{\partial x}+\frac{\partial v}{\partial y}+\frac{\partial w}{\partial z}
\end{aligned}
$$

Consider the following assumptions for the model:

- the system is in steady state (independent of $t$ and $\frac{\partial}{\partial t}$ terms vanish)

- no flow in the $z$-direction and $z$ independence $(\mathbf{u}=(u(x, y), v(x, y), 0), \rho=\rho(x, y)$, and $P=P(x, y))$

- model blood as a Newtonian fluid (viscosity, $\mu$, is constant)

- external force applied only in the $y$-direction $\left(\mathbf{F}=\left(F_{x}, F_{y}, F_{z}\right)=(0, \rho g, 0)\right)$

With these assumptions, together with the substitution of the appropriate components of the stress tensor (2.8) - (2.14), a simplified system of the conservation of momentum equations in component form is obtained, namely

$$
\begin{aligned}
& \rho\left(u u_{x}+v u_{y}\right)=\frac{1}{3} \mu \frac{\partial}{\partial x}\left(u_{x}+v_{y}\right)+\mu\left(u_{x x}+u_{y y}\right)-\frac{\partial P}{\partial x} \\
& \rho\left(u v_{x}+v v_{y}\right)=\frac{1}{3} \mu \frac{\partial}{\partial y}\left(u_{x}+v_{y}\right)+\mu\left(v_{x x}+v_{y y}\right)-\frac{\partial P}{\partial y}+\rho g
\end{aligned}
$$


where the $z$-momentum equation is identically satisfied.

Next, following Forrester and Young [9], assume that the constriction is mild. Two additional results follow from this assumption. First, equation (2.15) can be approximated as $\frac{\partial P}{\partial x}=0$. From the equation of state (2.4) this implies that $P=P(y)$ and $\rho=\rho(y)$.

With this result the conservation of mass equation (2.3) can be written as:

$$
\rho \frac{\partial u}{\partial x}+\frac{\partial(\rho v)}{\partial y}=0
$$

Second, as the fluid flows through the mild constriction we assume that the speed in the $x$-direction is negligible compared to the $y$-direction. Additionally, considering the flow in the $y$-direction, it can be assumed that changes in the $x$-direction are negligible compared to the $y$-direction. This implied that $u v_{x} \ll v v_{y}$ and we can neglect $u v_{x}$.

Using these two results we can write the system (2.15)-(2.16) as a single PDE in terms of $v(x, y)$, $\rho(y)$, and $P(y)$ :

$$
\rho v \frac{\partial v}{\partial y}=\frac{1}{3} \mu \frac{\partial}{\partial y}\left(\frac{-v}{\rho} \frac{d \rho}{d y}\right)+\mu\left(\frac{\partial^{2} v}{\partial x^{2}}+\frac{\partial^{2} v}{\partial y^{2}}\right)-\frac{d P}{d y}+\rho g
$$

Note: Equation (2.17) has been used to replace the $u_{x}+v_{y}$ in the first term of the right hand side of $(2.16)$.

In Chapter 3, the equation of state (2.4) will be used to write all pressure terms as density terms. Then, the analytic methods used to transform (2.18) into a differential equation in terms of $\frac{d \rho}{d y}$ will be discussed. 


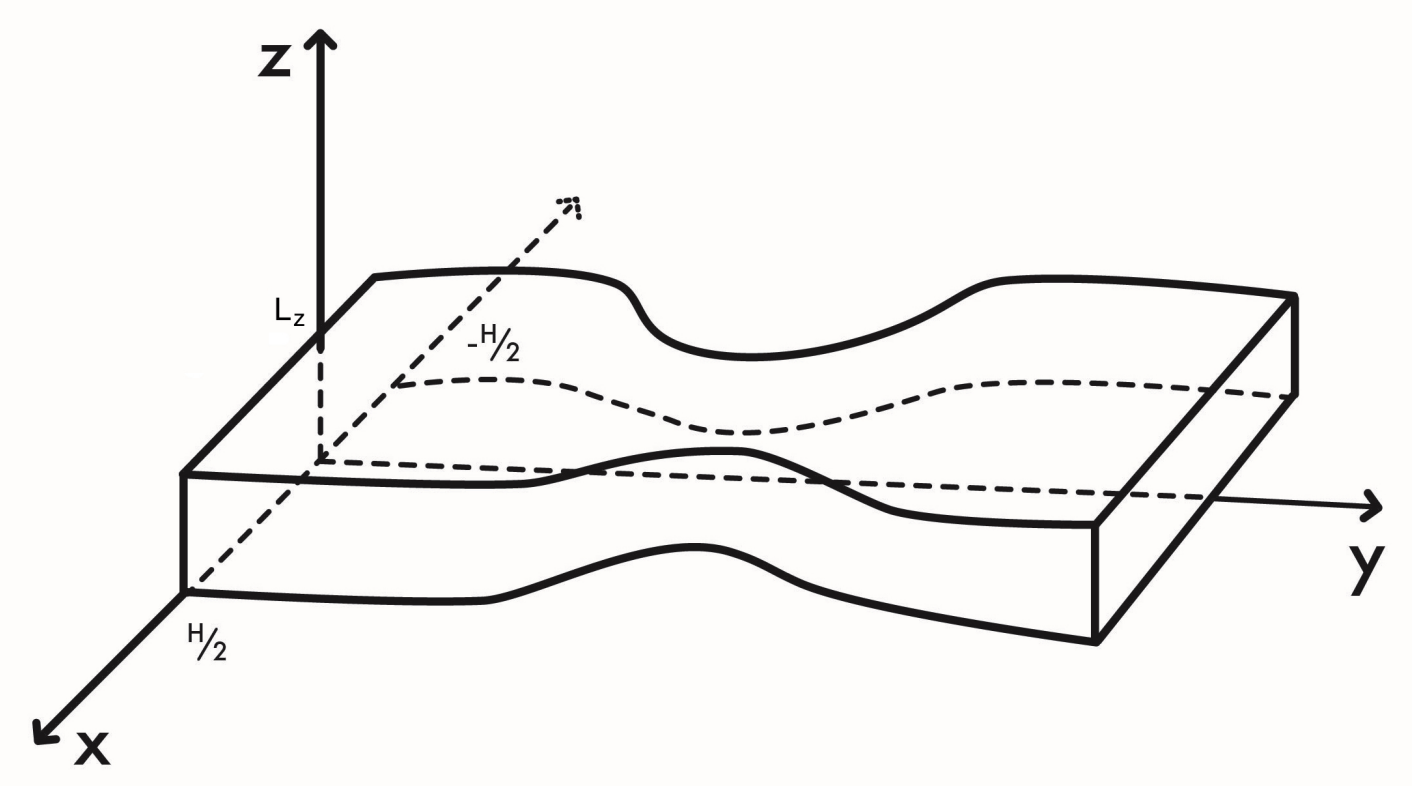

Figure 2.1: Flow geometry modelled as a rectangular microchannel.

\subsection{Flow Geometry}

The flow geometry for a constricted vessel is often modelled either experimentally ([10], [4], [6]) or theoretically $([3],[1],[24])$ as an axisymmetrical cylindrical tube. For this thesis, a rectangular geometry is chosen to model the constriction instead of the cylindrical geometry. This choice is largely motivated by the opportunity to compare our numerical results with experimental data. A team of engineers plans to construct a rectangular microchannel to further study the dynamics of blood-like fluids moving through a constriction. Figure 2.1 presents the general model of the flow domain. Assume that the channel is tall enough, that is $L_{z}$ large enough, for $z$-independence.

For the analysis, a two-dimensional cross section in the $x y$-plane of the channel is considered. A continuous, symmetric Gaussian distribution profile has been chosen for the boundary. Note: the 
cross section has been reflected, and the positive $x$-axis is directed up. This choice does not affect the model due to the symmetry. The distance, $R$, from the center line of the channel, is defined by:

$$
R(y)=R_{0}\left(1-\delta e^{-b y^{2}}\right)
$$

where $R_{0}$ is the unconstricted, constant, upstream and downstream distance from the centre of the channel, having units $m, \delta$ is a dimensionless quantity that determines the severity of the constriction, and $b$ determines the length of the constriction having units $m^{-2}$. This is shown in Figure 2.2. 


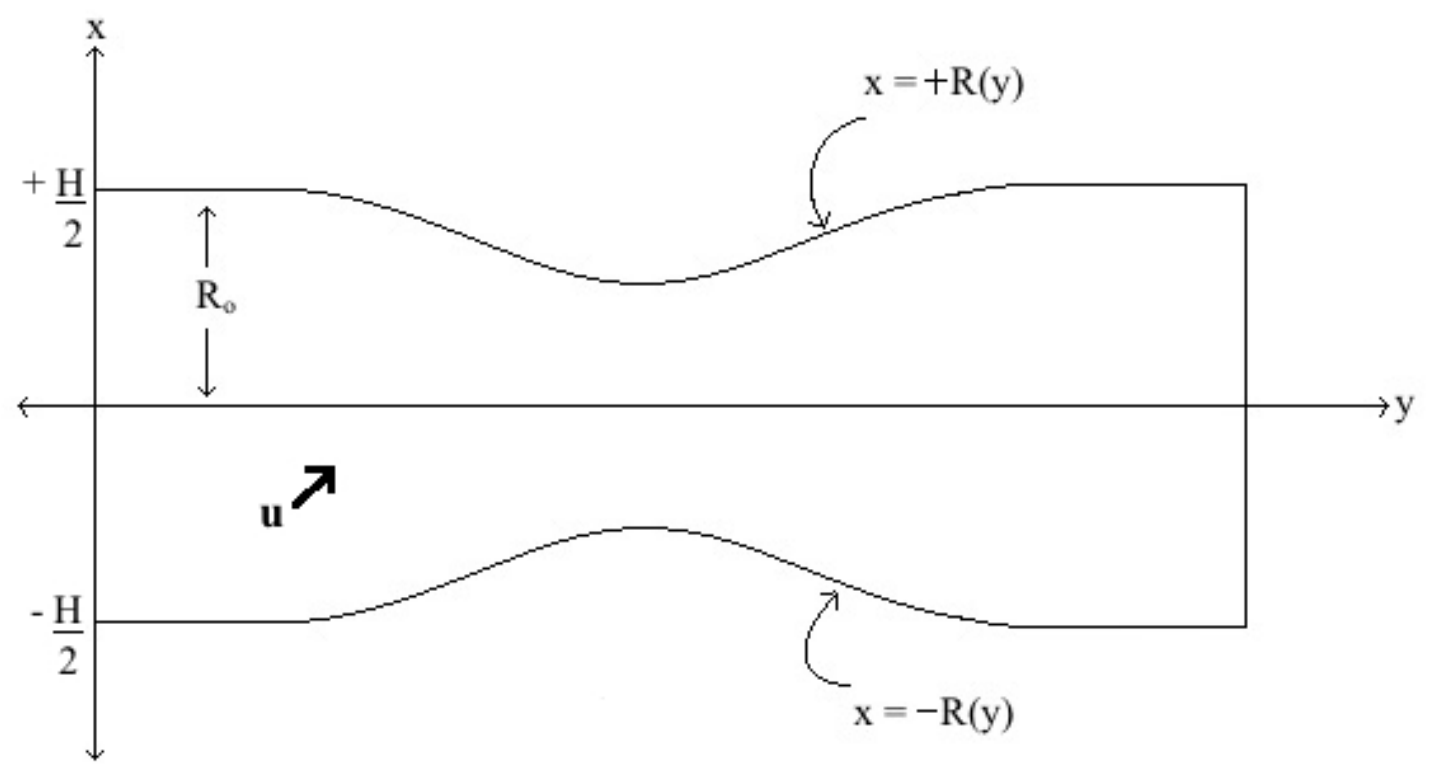

Figure 2.2: Cross-section of flow domain using Gaussian distribution profile for the boundary. 


\section{Chapter 3}

\section{Analytic Methods}

\subsection{The Assumed Solution for Velocity}

Following the work of Forrester and Young [9], begin by using the Karman-Pohlhausen method. Assume that the velocity profile in the $y$-direction is some fourth-order polynomial of the form:

$$
\frac{v}{V}=A \eta+B \eta^{2}+C \eta^{3}+D \eta^{4}+E
$$

where $\eta=\frac{R(y)-|x|}{R(y)}$ and $V=V(y)$ is the unknown centerline velocity, still to be determined. Note: With this definition, $v=v(x, y)$ is still maintained.

The following conditions arise from the model:

(i) $v=\frac{v_{s}}{\sqrt{1+R^{\prime 2}}}$ at $x= \pm R(y)$ (slip boundary condition)

(ii) $\frac{\partial v}{\partial x}=0$ at $x=0$ (approximate symmetric flow)

(iii) $v=V$ at $x=0$ (definition of centerline velocity)

(iv) $\frac{\partial^{2} v}{\partial x^{2}}=\frac{-8\left(V-v_{s}\right)}{H^{2}}$ at $x=0$ (nearly parabolic flow with slip)

(v) $\frac{d P}{d y} \approx \rho g+\mu v_{x x}$ at $x= \pm R(y)$ (approximation of (2.18) at the boundary) 
More specifically, condition (i) follows from solving the system $\mathbf{u} \cdot \mathbf{n}=0$ (the normal component of the velocity vanishes at the boundary) and $\mathbf{u} \cdot \mathbf{t}=v_{s}$ (the tangential component of the velocity has a magnitude of $v_{s}$ at the boundary). Conditions (ii) and (iv) come from assuming the velocity profile is nearly parabolic at the centre of the channel. Condition (iii) is the definition of the centerline velocity. Finally, condition $(\mathrm{v})$ is an approximation of the $y$-momentum equation at the boundary. The coefficients A - E in equation (3.1) can be determined by imposing these five conditions. This leads to,

$$
\begin{aligned}
A & =\frac{1}{6}(12-\lambda+T+2 M-12 E), \\
B & =\frac{1}{6}(3 \lambda-3 T), \\
C & =\frac{1}{6}(-12-3 \lambda+3 T-6 M+12 E), \\
D & =\frac{1}{6}(6+\lambda-T+4 M-6 E), \\
E & =\frac{v_{s}}{V \sqrt{1+R^{\prime 2}}}
\end{aligned}
$$

where,

$$
\begin{aligned}
& \lambda=\frac{R^{2}}{\mu V} \frac{d P}{d y} \\
& T=\frac{R^{2} \rho g}{\mu V} \\
& M=\frac{-4 R^{2}}{H^{2}} \frac{\left(V-v_{s}\right)}{V}
\end{aligned}
$$

Each expression (3.2)-(3.6) is dependent on $y$ only. Before an explicit expression for the centerline velocity can be determined, properties of the flow rate will be presented. 


\subsection{Flow Rate Analysis}

The mass flow rate, defined for the rectangular geometry, takes the form:

$$
\begin{aligned}
Q & =\int_{0}^{L_{z}} \int_{-R}^{R} \rho v d x d z \\
& =2 L_{z} \int_{0}^{R} \rho v d x
\end{aligned}
$$

where $L_{z}$ is the height of the channel in the $z$-direction and $R=R(y)$ is the width of the channel.

Two results follow from this definition. First, it will be shown that the change in mass flow rate in the direction of flow is zero. This implies that the flow rate is constant across the channel.

$$
\begin{array}{rlr}
\frac{d Q}{d y} & =\frac{d}{d y}\left(2 L_{z} \int_{0}^{R} \rho v d x\right) \\
& =2 L_{z} \frac{d}{d y}\left(\int_{0}^{R} \rho v d x\right) \\
& =2 L_{z}\left(\frac{\rho R^{\prime} v_{s}}{\sqrt{1+R^{\prime 2}}}+\int_{0}^{R} \frac{\partial}{\partial y}(\rho v) d x\right) & \quad \text { (using (A.3)) } \\
& =2 L_{z}\left(\frac{\rho R^{\prime} v_{s}}{\sqrt{1+R^{\prime 2}}}-\int_{0}^{R} \rho \frac{\partial u}{\partial x} d x\right) & \quad \text { (substitute }(2.17)) \\
& =2 L_{z}\left(\frac{\rho R^{\prime} v_{s}}{\sqrt{1+R^{\prime 2}}}-\left.\rho u\right|_{x=0} ^{x=R}\right) \\
& \left.=2 L_{z}\left(\frac{\rho R^{\prime} v_{s}}{\sqrt{1+R^{\prime 2}}}-\rho\left(\frac{R^{\prime} v_{s}}{\sqrt{1+R^{\prime 2}}}\right)\right) \quad \text { (from boundary condition and }\left.u\right|_{x=0}=0\right) \\
& =0
\end{array}
$$

Second, a relationship between the centerline velocity, $V$, and average velocity, $\bar{V}$, will be derived. Alternatively, the mass flow rate (3.10) can be defined in terms of the average velocity at any cross section along the channel. 


$$
Q=2 L_{z} \rho R \bar{V}
$$

Equating (3.10) and (3.12) and using the assumed solution (3.1) leads to the following relationship:

$$
\bar{V}=\frac{1}{R} \int_{0}^{R} V\left(A \eta+B \eta^{2}+C \eta^{3}+D \eta^{4}+E\right) d x
$$

Carrying out the integration and recalling that only $\eta$ is a function of $x$, leads to the expression:

$$
V=\frac{1}{\left(7 H^{2}-2 R^{2}\right)}\left(-\frac{1}{12} \frac{R^{2} H^{2} \rho g}{\mu}+\frac{1}{12} \frac{R^{2} H^{2}}{\mu} \frac{d P}{d y}-2 R^{2} v_{s}-3 \frac{v_{s} H^{2}}{\sqrt{1+R^{\prime 2}}}+10 H^{2} \bar{V}\right)
$$

The details of this calculation are presented in Appendix A.2.

These two results will be used in the next section to help derive a differential equation in terms of $\frac{d \rho}{d y}$ for the fluid as it moves through the constriction.

\subsection{The Density Gradient}

In this section the steps taken to transform the momentum PDE developed in Section 2.1 into an ODE in terms of $\frac{d \rho}{d y}$ are presented. Recall equation (2.18), namely:

$$
\rho v \frac{\partial v}{\partial y}=\rho g-\frac{d P}{d y}+\frac{\mu}{3} \frac{\partial}{\partial y}\left(\frac{-v}{\rho} \frac{d \rho}{d y}\right)+\mu\left(\frac{\partial^{2} v}{\partial x^{2}}+\frac{\partial^{2} v}{\partial y^{2}}\right)
$$

Begin by integrating across the channel. Since the boundary is symmetrical about the axis, bounds of integration can be changed to go from 0 to $R(y)$. 


$$
\int_{0}^{R}\left(\rho v \frac{\partial v}{\partial y}\right) d x=\int_{0}^{R}\left(\rho g-\frac{d P}{d y}+\frac{\mu}{3} \frac{\partial}{\partial y}\left(\frac{-v}{\rho} \frac{d \rho}{d y}\right)+\mu\left(\frac{\partial^{2} v}{\partial x^{2}}+\frac{\partial^{2} v}{\partial y^{2}}\right)\right) d x
$$

Since $\rho=\rho(y)$ and $P=P(y)$, the first two terms on the right side of the equation integrate easily. Also the integrand on the left side can be rewritten using the following substitution: $v \frac{\partial v}{\partial y}=$ $\frac{1}{2} \frac{\partial}{\partial y}\left(v^{2}\right)$.

$$
\begin{aligned}
\frac{1}{2} \rho \int_{0}^{R} \frac{\partial}{\partial y}\left(v^{2}\right) d x & =R \rho g-R \frac{d P}{d y}+\frac{\mu}{3} \int_{0}^{R}\left(\frac{\partial}{\partial y}\left(\frac{-v}{\rho} \frac{d \rho}{d y}\right)\right) d x \\
& +\mu \int_{0}^{R}\left(\frac{\partial^{2} v}{\partial x^{2}}\right) d x+\mu \int_{0}^{R}\left(\frac{\partial^{2} v}{\partial y^{2}}\right) d x
\end{aligned}
$$

Use the chain rule to expand the third term on the right hand side of the equation. This leads to:

$$
\begin{aligned}
\frac{1}{2} \rho \int_{0}^{R} \frac{\partial}{\partial y}\left(v^{2}\right) d x & =R \rho g-R \frac{d P}{d y}+\frac{\mu}{3} \frac{1}{\rho^{2}}\left(\frac{d \rho}{d y}\right)^{2} \int_{0}^{R} v d x \\
& -\frac{\mu}{3} \frac{1}{\rho}\left(\frac{d \rho}{d y}\right) \int_{0}^{R} \frac{\partial v}{\partial y} d x-\frac{\mu}{3} \frac{1}{\rho}\left(\frac{d^{2} \rho}{d y^{2}}\right) \int_{0}^{R} v d x \\
& +\mu \int_{0}^{R} \frac{\partial^{2} v}{\partial x^{2}} d x+\mu \int_{0}^{R} \frac{\partial^{2} v}{\partial y^{2}} d x
\end{aligned}
$$

Using the integral properties in Appendix A.1, the partial derivatives with respect to $y$ can be moved outside the integrals on both the left and right sides of equation (3.18). 


$$
\begin{aligned}
\frac{\rho}{2} \frac{d}{d y} \int_{0}^{R} v^{2} d x-\frac{\rho}{2} \frac{v_{s}^{2} R^{\prime}}{1+R^{\prime 2}} & =R \rho g-R \frac{d P}{d y}+\frac{\mu}{3} \frac{1}{\rho^{2}}\left(\frac{d \rho}{d y}\right)^{2} \int_{0}^{R} v d x-\frac{\mu}{3} \frac{1}{\rho} \frac{d \rho}{d y} \frac{d}{d y} \int_{0}^{R} v d x \\
& +\frac{\mu}{3} \frac{1}{\rho} \frac{d \rho}{d y} \frac{v_{s} R^{\prime}}{\sqrt{1+R^{\prime 2}}}+\mu \int_{0}^{R} \frac{\partial^{2} v}{\partial x^{2}} d x-\frac{\mu}{3} \frac{1}{\rho} \frac{d^{2} \rho}{d y^{2}} \int_{0}^{R} v d x \\
& +\mu \frac{d^{2}}{d y^{2}} \int_{0}^{R} v d x+\frac{2 \mu v_{s}\left(R^{\prime}\right)^{2}}{\left(1+R^{\prime 2}\right)^{\frac{3}{2}}} \frac{d^{2} R}{d y^{2}}-\frac{\mu v_{s}}{\sqrt{1+R^{\prime 2}}} \frac{d^{2} R}{d y^{2}}
\end{aligned}
$$

Now the flow rate relationship, $Q=2 L_{z} \rho \int_{0}^{R} v d x$, can be used to rewrite each of the integrals of $v$ in terms of $Q$ on the right hand side of the equation.

$$
\begin{aligned}
\frac{\rho}{2} \frac{d}{d y} \int_{0}^{R} v^{2} d x & =R \rho g-R \frac{d P}{d y}+\frac{\rho}{2} \frac{v_{s}^{2} R^{\prime}}{1+R^{\prime 2}}+\frac{\mu}{3} \frac{1}{\rho^{2}}\left(\frac{d \rho}{d y}\right)^{2}\left[\frac{Q}{2 L_{z} \rho}\right]-\frac{\mu}{3} \frac{1}{\rho} \frac{d \rho}{d y} \frac{d}{d y}\left[\frac{Q}{2 L_{z} \rho}\right] \\
& -\frac{\mu}{3} \frac{1}{\rho} \frac{d^{2} \rho}{d y^{2}}\left[\frac{Q}{2 L_{z} \rho}\right]+\frac{\mu}{3} \frac{1}{\rho} \frac{d \rho}{d y} \frac{v_{s} R^{\prime}}{\sqrt{1+R^{\prime 2}}}+\left.\mu\left(\frac{\partial v}{\partial x}\right)\right|_{x=R}+\mu \frac{d^{2}}{d y^{2}}\left[\frac{Q}{2 L_{z} \rho}\right] \\
& +\frac{2 \mu v_{s}\left(R^{\prime}\right)^{2}}{\left(1+R^{\prime 2}\right)^{\frac{3}{2}}} \frac{d^{2} R}{d y^{2}}-\frac{\mu v_{s}}{\sqrt{1+R^{\prime 2}}} \frac{d^{2} R}{d y^{2}}
\end{aligned}
$$

Note that the second partial derivative of $v$ with respect to $x$ on the right hand side has been integrated and the symmetric flow condition (ii), namely $\left.\frac{\partial v}{\partial x}\right|_{x=0}=0$, has been used. In Section 3.2 it was demonstrated that the flow rate, $Q$, is a constant. Differentiating the flow rate expressions on the right hand side, along with $\rho=\rho(y)$, leads to:

$$
\begin{aligned}
\frac{\rho}{2} \frac{d}{d y} \int_{0}^{R} v^{2} d x & =R \rho g-R \frac{d P}{d y}+\frac{\rho}{2} \frac{v_{s}^{2} R^{\prime}}{1+R^{\prime 2}}+\frac{\mu}{3} \frac{1}{\rho^{2}}\left(\frac{d \rho}{d y}\right)^{2}\left[\frac{Q}{2 L_{z} \rho}\right]+\frac{\mu}{3} \frac{1}{\rho} \frac{d \rho}{d y}\left[\frac{Q}{2 L_{z} \rho^{2}}\right] \frac{d \rho}{d y} \\
& -\frac{\mu}{3} \frac{1}{\rho} \frac{d^{2} \rho}{d y^{2}}\left[\frac{Q}{2 L_{z} \rho}\right]+\frac{\mu}{3} \frac{1}{\rho} \frac{d \rho}{d y} \frac{v_{s} R^{\prime}}{\sqrt{1+R^{\prime 2}}}+\mu\left[\frac{Q}{L_{z} \rho^{3}}\left(\frac{d \rho}{d y}\right)^{2}-\frac{Q}{2 L_{z} \rho^{2}} \frac{d^{2} \rho}{d y^{2}}\right] \\
& +\left.\mu\left(\frac{\partial v}{\partial x}\right)\right|_{x=R}+\frac{2 \mu v_{s}\left(R^{\prime}\right)^{2}}{\left(1+R^{\prime 2}\right)^{\frac{3}{2}}} \frac{d^{2} R}{d y^{2}}-\frac{\mu v_{s}}{\sqrt{1+R^{\prime 2}}} \frac{d^{2} R}{d y^{2}}
\end{aligned}
$$

Collect the like terms to simplify the right side of the equation. Then, use the equation of state 
(2.4) to write $P$ in terms of $\rho$. Refer to (A.13) for details pertaining to evaluating $\left.\left(\frac{\partial v}{\partial x}\right)\right|_{x=R}$.

$$
\begin{aligned}
\frac{\rho}{2} \frac{d}{d y} \int_{0}^{R} v^{2} d x & =R \rho g-R \frac{k_{B} T}{m} \frac{d \rho}{d y}+\frac{\rho}{2} \frac{v_{s}^{2} R^{\prime}}{1+R^{\prime 2}}+\frac{8 \mu}{3}\left(\frac{d \rho}{d y}\right)^{2}\left[\frac{Q}{2 L_{z} \rho^{3}}\right]-\frac{4 \mu}{3} \frac{d^{2} \rho}{d y^{2}}\left[\frac{Q}{2 L_{z} \rho^{2}}\right] \\
& +\frac{\mu}{3} \frac{1}{\rho} \frac{d \rho}{d y} \frac{v_{s} R^{\prime}}{\sqrt{1+R^{\prime 2}}}-\frac{\mu V A}{R}+\frac{2 \mu v_{s}\left(R^{\prime}\right)^{2}}{\left(1+R^{\prime 2}\right)^{\frac{3}{2}}} \frac{d^{2} R}{d y^{2}}-\frac{\mu v_{s}}{\sqrt{1+R^{\prime 2}}} \frac{d^{2} R}{d y^{2}}
\end{aligned}
$$

Use equation (3.12) to write $Q$ in terms of the average velocity, $\bar{V}$. Next, substitute the expression (A.14), derived in appendix, for the $\frac{V A}{R}$ term. The equation of state has been used to write $\frac{d P}{d y}$ in terms of $\frac{d \rho}{d y}$. Collecting like terms and simplifying leads to:

$$
\begin{aligned}
\frac{\rho}{2} \frac{d}{d y} \int_{0}^{R} v^{2} d x & =\frac{5}{6} R \rho g-\frac{5}{6} R \frac{k_{B} T}{m} \frac{d \rho}{d y}+\frac{\rho}{2} \frac{v_{s}^{2} R^{\prime}}{1+R^{\prime 2}}+\frac{8}{3} \frac{\mu R \bar{V}}{\rho^{2}}\left(\frac{d \rho}{d y}\right)^{2}-\frac{4}{3} \frac{\mu R \bar{V}}{\rho} \frac{d^{2} \rho}{d y^{2}} \\
& +\frac{\mu}{3} \frac{1}{\rho} \frac{d \rho}{d y} \frac{v_{s} R^{\prime}}{\sqrt{1+R^{\prime 2}}}-\frac{2 \mu V}{R}+\frac{4}{3} \frac{\mu V R}{H^{2}}-\frac{4}{3} \frac{\mu v_{s} R}{H^{2}}-\frac{2 \mu v_{s}}{R \sqrt{1+R^{\prime 2}}} \\
& +\frac{2 \mu v_{s}\left(R^{\prime}\right)^{2}}{\left(1+R^{\prime 2}\right)^{\frac{3}{2}}} \frac{d^{2} R}{d y^{2}}-\frac{\mu v_{s}}{\sqrt{1+R^{\prime 2}}} \frac{d^{2} R}{d y^{2}}
\end{aligned}
$$

At this point Maple is used to evaluate the expression on the left side of (3.23). The details in obtaining an ODE in terms of $\frac{d \rho}{d y}$ are as follows:

- Integrate $\int_{0}^{R} v^{2} d x$ using the assumed solution from Section 3.1.

- Differentiate the resulting expression with respect to $y$. Refer to (A.12) for the derivative of $V(y)$.

- Using the equation of state, (2.4), substitute all $P$ terms for expressions with $\rho$.

- Non-dimensionalize the final equation by dividing each term by the factor $\frac{\mu \bar{V}}{R}$.

- Introduce two dimensionless quantities: 
- Mach Number:

$$
M a=\frac{\bar{V}}{\sqrt{\frac{k_{B} T}{m}}}
$$

- Reynolds Number:

$$
R e=\frac{\rho \bar{V} R}{\mu}
$$

- Collect terms with respect to $\frac{d \rho}{d y}$ to reveal structure of ODE

After following those steps a second-order, nonlinear ODE is obtained with the general form:

$$
0=\Omega_{1}\left(\frac{d^{2} \rho}{d y^{2}}\right)+\Omega_{2}\left(\frac{d \rho}{d y}\right)^{2}+\Omega_{3}\left(\frac{d \rho}{d y}\right)+\Omega_{4}
$$

where the coefficients $\Omega_{1}, \Omega_{2}, \Omega_{3}$, and $\Omega_{4}$ are non-constant coefficients dependent on $y$. More specifically, they contain $\rho=\rho(y), \bar{V}=\bar{V}(y)$, and $R=R(y)$ terms as well as the dimensionless quantities $R e=\operatorname{Re}(y)$ and $M a=M a(y)$. It is important to point out that some terms in $\Omega_{1}$ also depend on $\frac{d \rho}{d y}$. The specific ODE is presented in Appendix A.6.

\subsection{Upstream Analysis}

In the current form, equation (3.26) cannot be solved numerically. The $\bar{V}(y), \operatorname{Re}(y)$, and $M a(y)$ terms need to be written in terms of $\rho$ and $R$. Recall that the flow rate is constant through out the channel, as shown in Section 3.2. From (3.12) it follows that:

$$
2 L_{z} \rho R \bar{V}=2 L_{z} \rho_{0} R_{0} \bar{V}_{0}
$$


where $\rho_{0}, R_{0}$, and $\bar{V}_{0}$ represent constant upstream values of the density, channel width, and average velocity respectively. Using (3.27), along with the definition of the Reynolds number (3.25) and the Mach number (3.24), leads to:

$$
\begin{aligned}
& \bar{V}=\frac{\rho_{0} R_{0}}{\rho R} \bar{V}_{0} \\
& R e=R e_{0} \\
& M a=\frac{\rho_{0} R_{0}}{\rho R} M a_{0}
\end{aligned}
$$

where $R e_{0}$ and $M a_{0}$ represent upstream values of the Reynolds and Mach numbers. Notice that this implies the Reynolds number is constant throughout the entire channel.

From an additional analysis, the following relationship holds true:

$$
R e=\frac{3\left(1-v_{s_{0}}\right)}{g_{0}}
$$

where $v_{s_{0}}$ is the scaled, upstream magnitude of the slip velocity and $g_{0}=\frac{g R_{0}}{\bar{V}_{0}^{2}}$ is the scaled, upstream force term. The details of this calculation are presented in Appendix A.5. With these relationships, a numerical solution to $(3.26)$ can be determined. 


\section{Chapter 4}

\section{Numerical Solutions}

In this chapter the numerical solutions to the second order, non-linear differential equation derived in the previous chapter are presented. Recall, the ODE has the form:

$$
0=\Omega_{1}\left(\frac{d^{2} \rho}{d y^{2}}\right)+\Omega_{2}\left(\frac{d \rho}{d y}\right)^{2}+\Omega_{3}\left(\frac{d \rho}{d y}\right)+\Omega_{4}
$$

The Runge-Kutta fourth-order method in Maple is used to determine numerical solutions for $\rho(y)$ and $\frac{d \rho}{d y}$. In the following sections the role of the slip velocity, compressibility, and the geometry of the constriction, and Reynolds number are investigated. Also investigated is the role of the second-order derivative and the solution is compared with the results presented in ([24], [20]). Let $\mu=0.0035 \frac{\mathrm{kg}}{\mathrm{m} \cdot \mathrm{s}}$ for consistency with $([24],[20])$. From the upstream analysis in Section 3.4, the Reynolds Number, Mach Number, and average velocity can be written in terms of independently chosen upstream values. For the results presented below, the initial values $\rho_{0}=20 \frac{\mathrm{kg}}{\mathrm{m}^{3}}, \overline{V_{0}}=$ $0.4725 \frac{\mathrm{m}}{\mathrm{s}}, g_{0}=0.01, v_{s 0}=0.1, R e=270$ and $M a_{0}=0.1$ have been chosen. Recall that the constriction was modelled using a Gaussian profile distribution. For this Thesis, $R_{0}=0.1 \mathrm{~m}$, $\delta=0.25$, and $b=25 m^{-2}$ were chosen as the standard geometry. These values can be changed to correspond to an experimental domain in later research. 


\subsection{Role of Slip Velocity}

We present solution curves for varying slip velocities. It is important to note that in changing the value of the slip velocity, the Reynolds number also changes according to equation (3.31).

Figure 4.1 and Figure 4.2 show the solutions for the centerline density and the density gradient while varying $v_{s_{0}}$. The upstream slip velocities of $v_{s_{0}}=0,0.1,0.5,0.9,0.95$ were considered. The solid line solutions correspond to the standard choice stated above, with $v_{s_{0}}=0.1$, and will serve as the reference graph. In the no slip case, $v_{s_{0}}=0$, the decrease in centerline density is slightly less compared to the case when $v_{s_{0}}=0.1$ as it moves through the constriction. However, the post constriction density for the no slip case remains slightly less than the $v_{s_{0}}=0.1$ case. More generally, Figure 4.1 shows that as the slip velocity increases, there is a greater decrease in centerline density through the constriction. This observation is supported by the increasing magnitude of the preconstriction maxima in Figure 4.2. The figures also suggest that as the slip velocity increases, the post-constriction density recovers to smaller values for $v_{s_{0}} \geq 0.5$. These trends seem to agree with the results presented in [20]. It is important to note that as the upstream $v_{s 0} \rightarrow 1$, the Reynolds number approaches zero from equation (3.31).

Interestingly, when $v_{s 0}=0.5$, the post constriction recovery occurs at a faster rate compared to $v_{s 0}=0$ and $v_{s 0}=0.1$, but settles to roughly the same downstream density. The maxima in Figure 4.2 show that as $v_{s 0}$ increases the changes in pre-constriction density also increase. Also, the asymmetry in the density gradient curves indicate that the change in post-constriction density is less than the changes in pre-constriction density.

\subsection{Role of Compressibility}

Blood is weakly compressible by nature and research has shown that the effects of compressibility should not be ignored ([3], [19]). An incompressible fluid is modelled using $M a=0$ and increasing the Mach number increases the compressibility of the fluid. Figure 4.3 and Figure 4.4 show the solutions for the centerline density and density gradient, respectively, while varying the Mach 
number using the values $M a=0.01,0.05,0.1,0.2$, and 0.35 . The solid line solutions correspond to the standard choice of $M a=0.1$ and will serve as the reference graph. Both figures suggest as $M a \rightarrow$ 0 the change in density through the constriction and post constriction is almost zero. However, Figure 4.3, suggests that increased compressibility of the fluid (i.e. larger Mach number values), correspond to greater decreases in the density through the constriction. This is also supported in Figure 4.4 by noting that the magnitude of the maxima increase with respect to increasing Mach number. Similar to slip velocity, increasing the Mach number corresponds to a decrease in downstream density. The solution diverges for $M a>0.5$.

Figure 4.5 and Figure 4.6 show the centerline density and density gradient solutions corresponding to zero slip for the same choices of Mach number as above. Note that $v_{s 0}=0$ corresponds to $R e=300$. These results suggest that there are no significant differences in the solutions for the cases $v_{s 0}=0$ and $v_{s 0}=0.1$. This was expected as a similar trend was demonstrated in [20].

\subsection{Role of Geometry}

In Chapter 2, the analysis of the Navier-Stokes equation began by following [9] and assuming a mild constriction in the flow domain. It is expected that the solution profile would be affected by the choice of the severity $(\delta)$ and the length $(b)$ of the constriction.

\subsubsection{Effects of varying $\delta$}

Figure 4.7 and Figure 4.8 show the centerline density and density gradient solutions, respectively, while varying the severity of the constriction. The values $\delta=0.05,0.1,0.25,0.45$ were chosen. The solid line solutions correspond to the standard choice of $\delta=0.25$ and will serve as the reference graph. These solutions show very similar characteristics to the solutions presented with varying the compressibility. As $\delta \rightarrow 0$ the solution approaches the constant solution observed for a channel with no constriction. As $\delta$ increases, there is an increase in the change in density through the constriction. Again, similar to Mach number and slip velocity, as $\delta$ is increased there is a decrease 
in the post constriction density. It was observed that for $\delta>0.49$ the solution diverges, which agrees with the original assumption of a mild constriction.

\subsubsection{Effects of varying $b$}

Figure 4.9 and Figure 4.10 show the centerline density and density gradient solutions, respectively, while varying the length of the constriction. The values $b=10,25,50,100$ were chosen. The solid line solutions correspond to the standard choice of $b=25$ and will serve as the reference graph. As $b$ decreases, there is a greater decrease in centerline density through the constriction. However, comparing Figure 4.10 with Figure 4.8 it can be seen that increasing $\delta$ has a greater affect on both the pre- and post-constriction density changes than decreasing $b$. It was also observed that for $b>112$ the solution diverges.

Figure 4.9 shows that the downstream centerline density recovers to similar values for the different choices of $b$. Also, Figure 4.10 shows that as $b$ decreases, the pre-constriction centerline density changes sooner and does not peak as high. Both these results are different than what was observed for varying values of $v_{s 0}, M a$, and $\delta$.

\subsection{Role of Reynolds Number}

The role of the Reynolds number was also investigated to compare with ([24], [20]). In Section 3.4, an expression for the upstream Reynolds number was developed, namely:

$$
R e=\frac{3\left(1-v_{s 0}\right)}{\frac{g R_{0}}{\bar{V}_{0}^{2}}}
$$

The Reynolds number, as defined in equation (3.25), can also be written in terms of upstream values as: 


$$
R e=\frac{\rho_{0} R_{0} \bar{V}_{0}}{\mu}
$$

Substituting equation (4.3) into equation (4.2) and solving for $\bar{V}_{0}$, leads to:

$$
\bar{V}_{0}=\frac{\rho_{0} R_{0}^{2} g}{3 \mu\left(1-v_{s 0}\right)}
$$

Equations (4.4) and (4.3) show that both the upstream Reynolds number and the upstream average velocity are proportional to the external force, $g$. By varying the values of $g$, different Reynolds numbers can be investigated without changing the initial values used in Sections 4.1 - 4.4.

Moderate to low Reynolds numbers were chosen which are more appropriate for microchannels. Low Reynolds numbers are also more appropriate when using MPC methods. Figure (4.11) and Figure (4.12) show the solutions for the centerline density and density gradient respectively for the values $R e=241,120,60$ and 12 . Figure 4.11 shows that as the Reynolds number decreases, there is a greater pre-constriction decrease in centerline density. Interestingly, for small Reynolds numbers $(R e \sim 12)$, there is a significant decrease in density as the fluid moves through the constriction, compared to the moderate Reynolds numbers. Also, the density does not increase post-constriction, rather is remains relatively constant. This trend is also shown in Figure 4.12.

\subsection{Role of second-order derivative}

In ([24], [20]), the first-order differential equation for the pressure gradient was solved numerically. This corresponds to the $\frac{d^{2} \rho}{d y^{2}}$ being dropped in (4.1). Figure 4.13 compares the first-order solution for density with the second-order solution with zero slip velocity on the boundary. Figure 4.14 compares the two solutions for density with slip velocity incorporated. The solid line solution corresponds to the second order DE solved in this Thesis and the dashed line corresponds to the 
first-order solution presented in [24].

Both figures suggest similar differences between the first and second order solutions. The inclusion of the second order term decreases the magnitude of the drop in density as the fluid moves through the constriction. Interestingly, for the second order solution, the minimum density is achieved at a position that is downstream of the maximum constriction. Additionally, Figure 4.14 suggests that when slip velocity is incorporated the difference between the minimum density and constant downstream density between the two solutions is less when compared with Figure 4.13 .

\subsection{Graphical Results}

The figures discussed in Sections 4.1 - 4.5 are presented. 


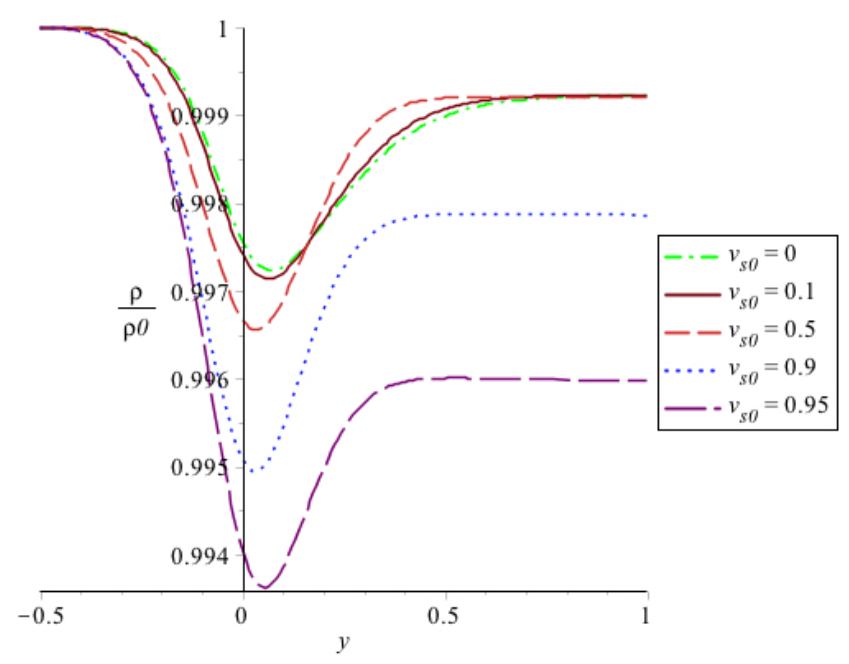

Figure 4.1: Solution for scaled density while varying slip velocity, $v_{s_{0}}$, with $M a_{0}=0.1$ and $R_{0}=$ $0.1 m, \delta=0.25, b=25 m^{-2}$.

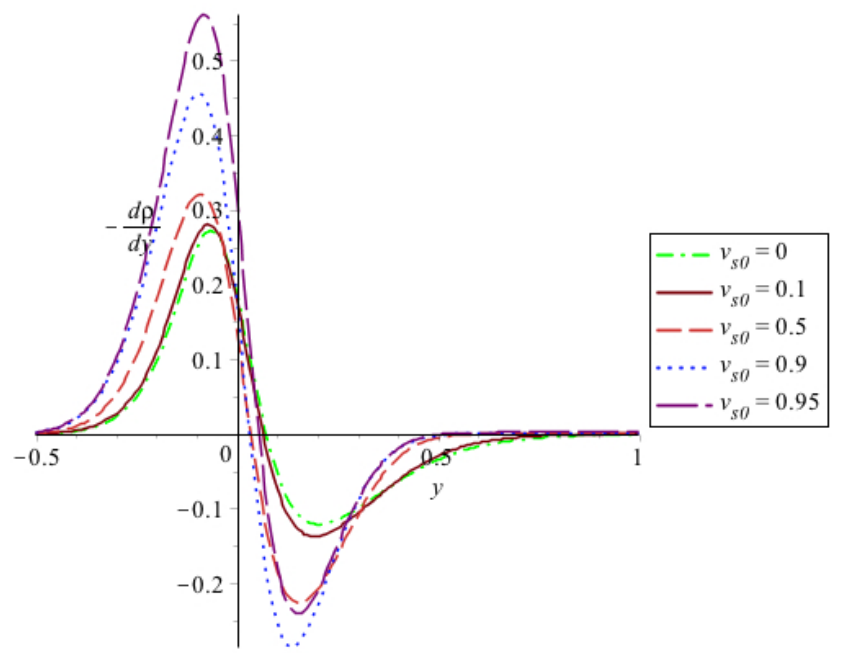

Figure 4.2: Solution for density gradient while varying slip velocity, $v_{s_{0}}$, with $M a_{0}=0.1$ and $R_{0}=0.1 m, \delta=0.25, b=25 m^{-2}$. 


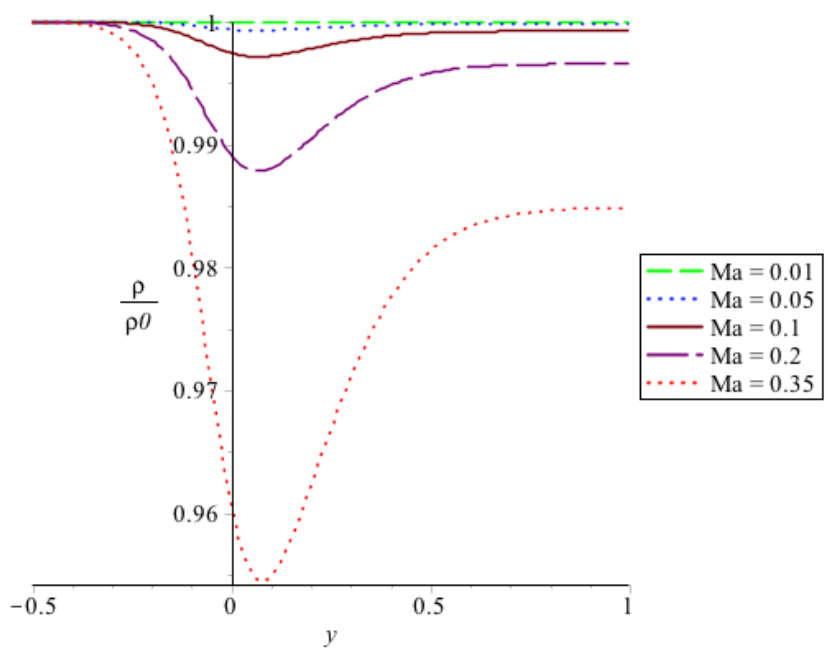

Figure 4.3: Solution for scaled density while varying Mach Number, $M a$, with $v_{s_{0}}=0.1$ and $R_{0}=0.1 m, \delta=0.25, b=25 m^{-2}$.

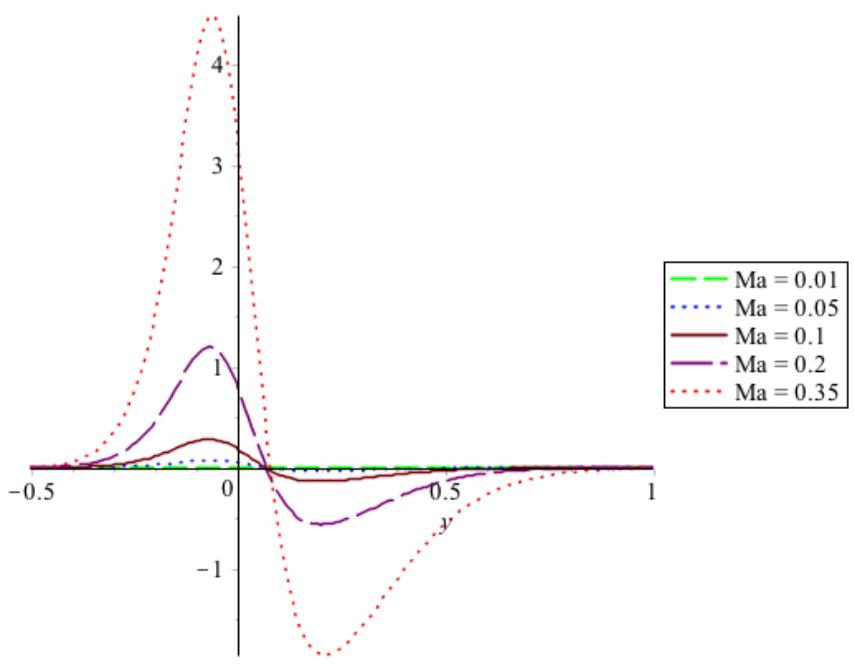

Figure 4.4: Solution for density gradient while varying Mach Number, $M a$, with $v_{s_{0}}=0.1$ and $R_{0}=0.1 m, \delta=0.25, b=25 m^{-2}$. 


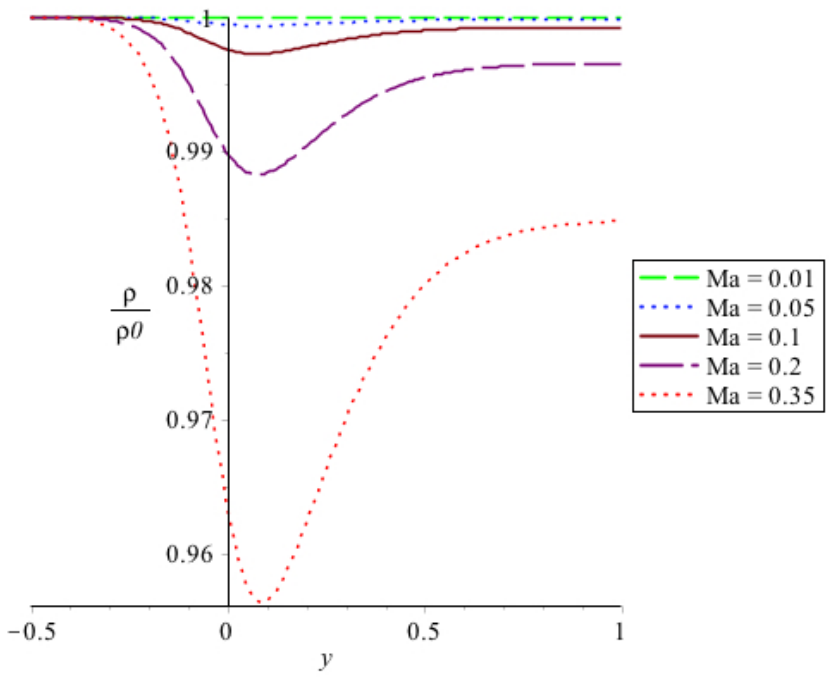

Figure 4.5: Solution for scaled density while varying Mach Number, $M a$, with $v_{s_{0}}=0$ and $R_{0}=$ $0.1 m, \delta=0.25, b=25 m^{-2}$.

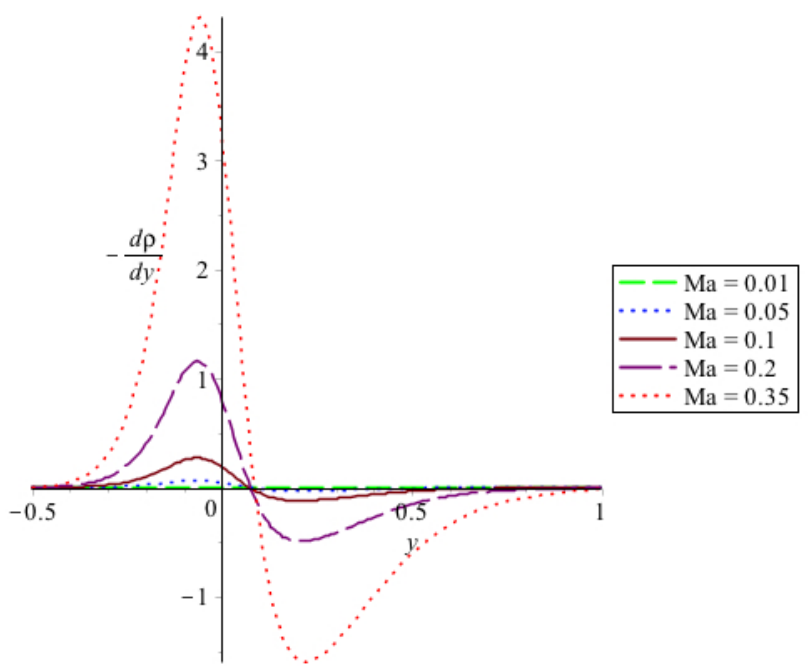

Figure 4.6: Solution for density gradient while varying Mach Number, $M a$, with $v_{s_{0}}=0$ and $R_{0}=0.1 m, \delta=0.25, b=25 m^{-2}$. 


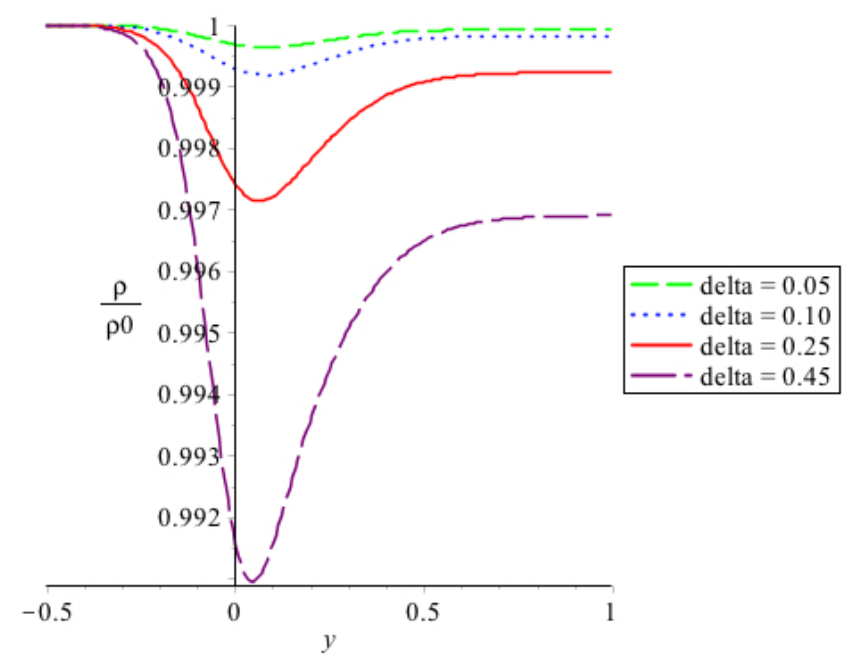

Figure 4.7: Solution for scaled density while varying severity of constriction, $\delta$, with with $M a_{0}=0.1$, $v_{s_{0}}=0.1, R e=270$ and $R_{0}=0.1 m, b=25 m^{-2}$.

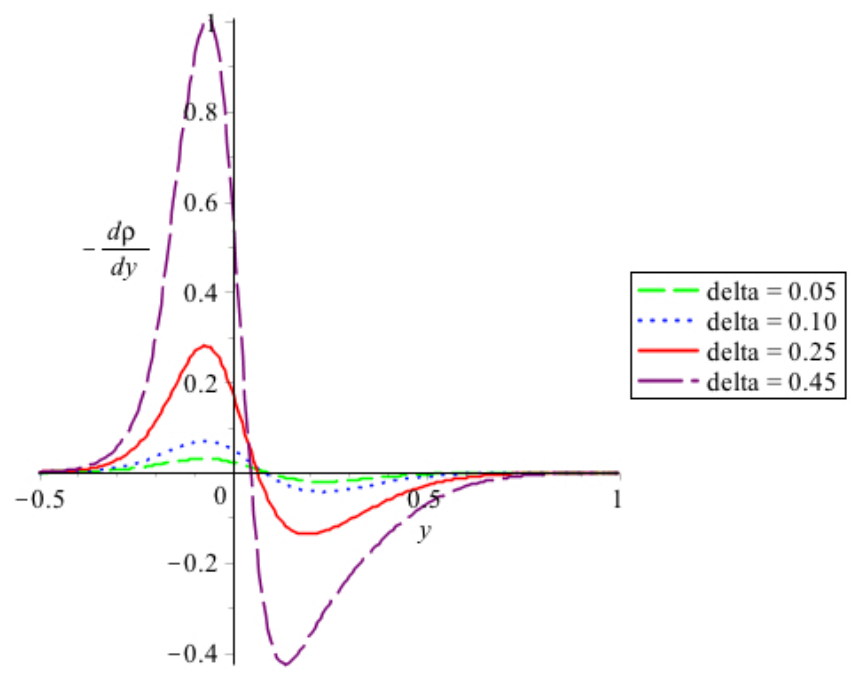

Figure 4.8: Solution for density gradient while varying varying severity of constriction, $\delta$, with $M a_{0}=0.1, v_{s_{0}}=0.1, R e=270$ and $R_{0}=0.1 \mathrm{~m}, b=25 \mathrm{~m}^{-2}$. 


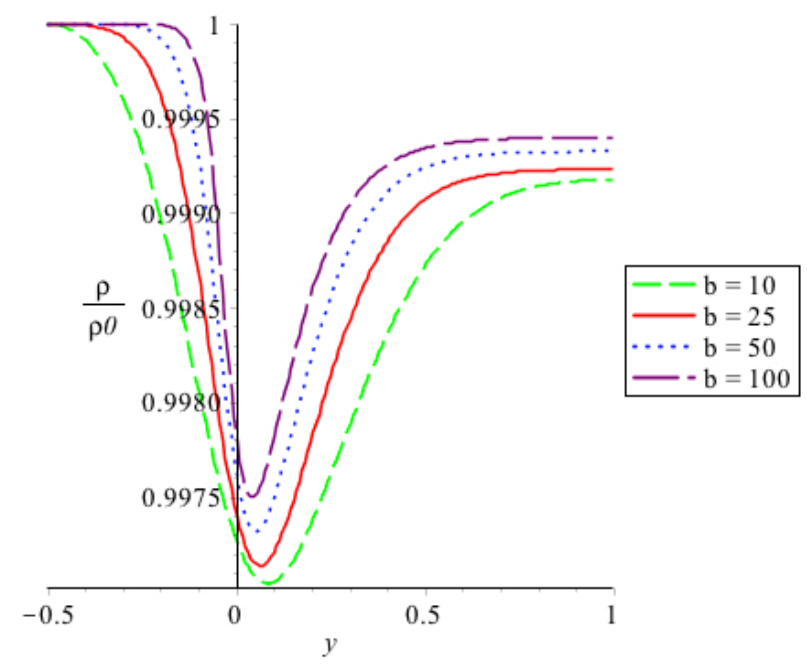

Figure 4.9: Solution for scaled density while varying width of constriction, $b$, with $M a_{0}=0.1$, $v_{s_{0}}=0.1, R e=270$ and $R_{0}=0.1 \mathrm{~m}, \delta=0.25 \mathrm{~m}^{-2}$.

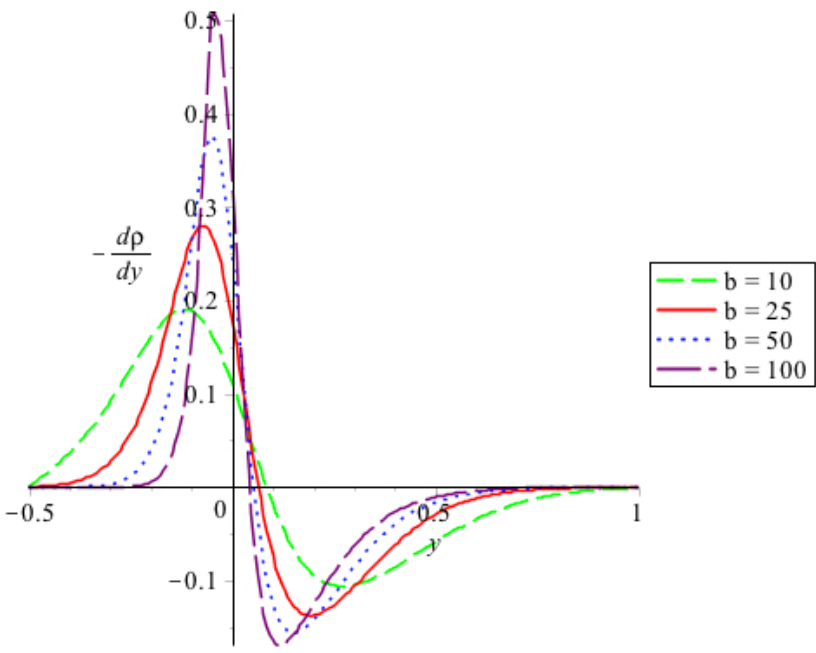

Figure 4.10: Solution for density gradient while varying width of constriction, $b$, with $M a_{0}=0.1$, $v_{s_{0}}=0.1, R e=270$ and $R_{0}=0.1 \mathrm{~m}, \delta=0.25 \mathrm{~m}^{-2}$. 


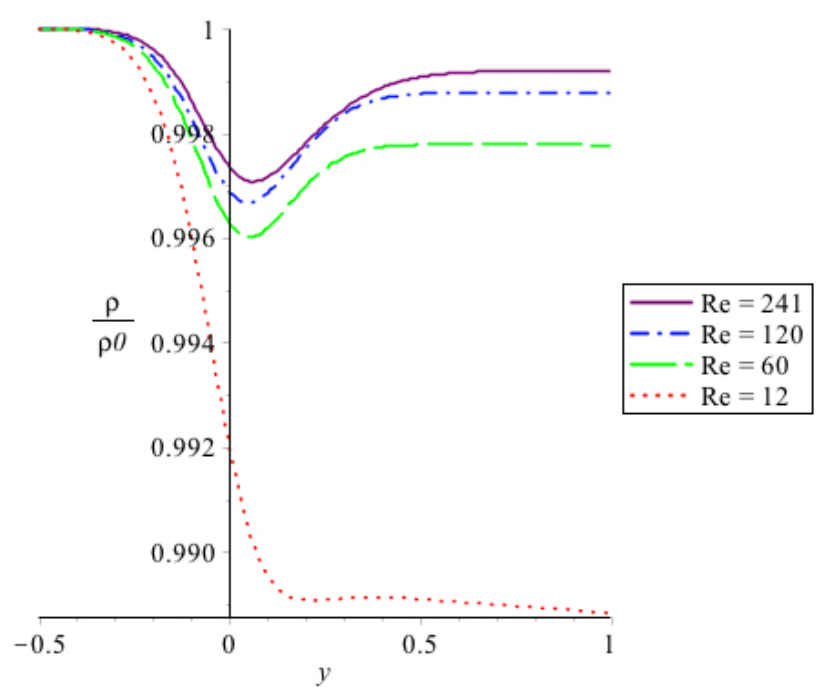

Figure 4.11: Solutions for scaled density while varying Reynolds number, $R e$, with $M a_{0}=0.1$, $v_{s_{0}}=0.1$, and $R_{0}=0.1 m, \delta=0.25, b=25 m^{-2}$.
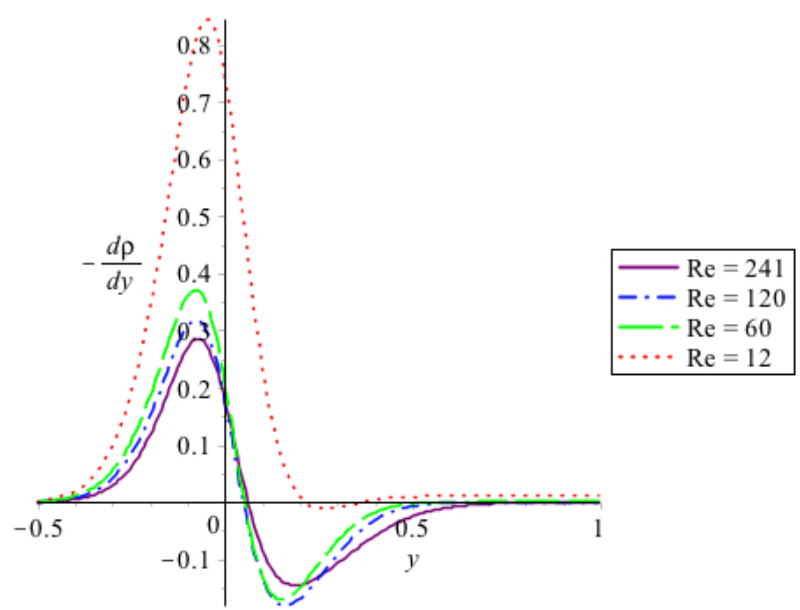

Figure 4.12: Solutions for density gradient while varying Reynolds number, $R e$, with $M a_{0}=0.1$, $v_{s_{0}}=0.1$, and $R_{0}=0.1 m, \delta=0.25, b=25 m^{-2}$. 


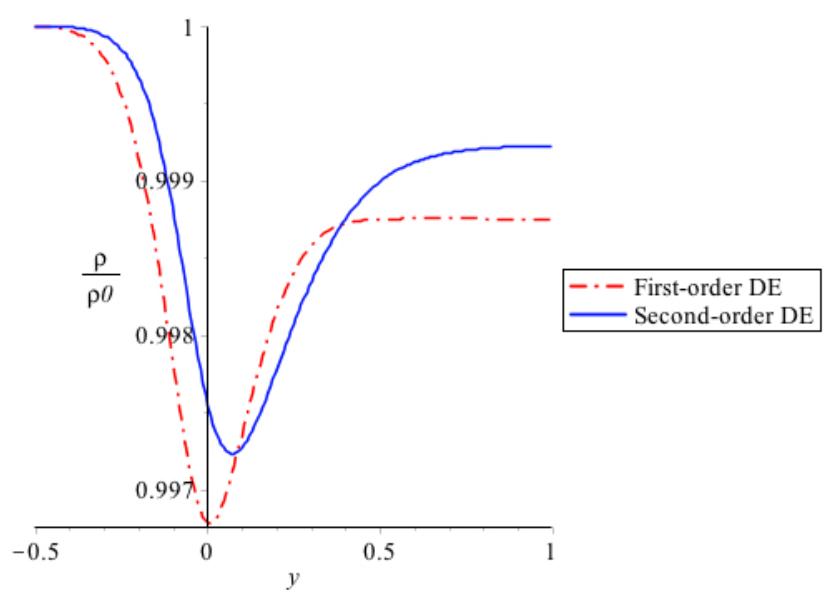

Figure 4.13: Solutions for scaled density for first order DE compared to second order DE with $M a_{0}=0.1, v_{s_{0}}=0, R e=300$ and $R_{0}=0.1 m, \delta=0.25, b=25 m^{-2}$.

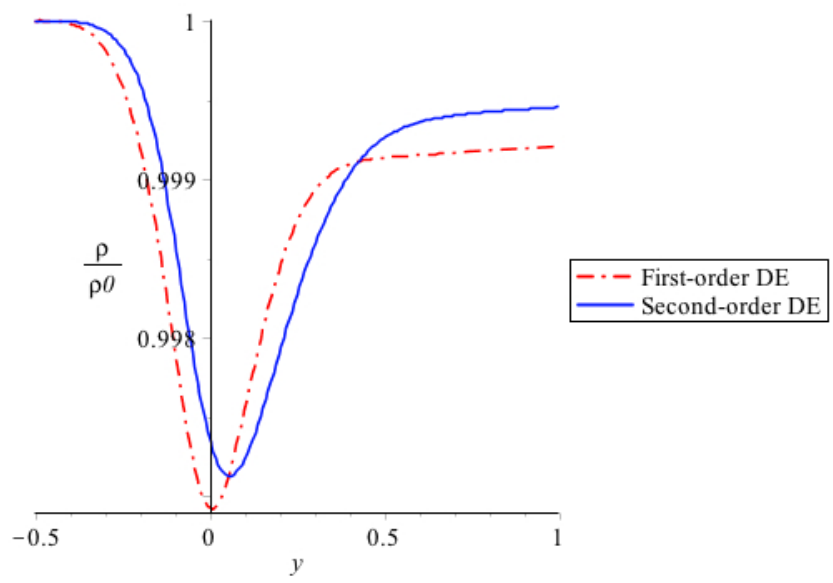

Figure 4.14: Solutions for scaled density for first order DE compared to second order DE with $M a_{0}=0.1, v_{s_{0}}=0.1, R e=270$ and $R_{0}=0.1 \mathrm{~m}, \delta=0.25, b=25 \mathrm{~m}^{-2}$. 


\section{Chapter 5}

\section{Conclusions and Future Work}

In this Thesis an approximate analytic solution for centerline density is developed for a weakly compressible fluid moving through a rectangular channel with a symmetrical mild constriction. The choice to use a rectangular channel was largely motivated by the opportunity to compare with experimental data that will further explore the dynamics of blood-like fluids. A smooth, continuous Gaussian distribution profile was chosen to model the constriction. In Chapter 3, the Karman-Pohlhausen method was used to transform the Navier-Stokes momentum equations into a single non-linear, second-order differential equation in terms of $\frac{d \rho}{d y}$.

Using the Runge-Kutta fourth-order method in Maple, numerical solutions were obtained and presented in Chapter 4. Parameters such as slip velocity, compressibility, geometry of the constriction, and Reynolds number were isolated to investigate their role in the flow properties of the fluid. It was found that varying the Mach number (i.e. compressibility) had a greater effect on the change in density, compared to variations in the slip velocity. It was also observed that incorporating slip while varying the Mach number had little to no affect on the solution curves.

Small values of the severity of the constriction, $\delta$ were chosen to agree with the earlier assumption that the constriction was mild. As the length of the constriction, $b$, was increased it was observed that the magnitude of the decrease in centerline density became smaller and decrease occurred over a greater distance. An unexpected result came from the Reynolds number analysis. While 
moderate Reynolds numbers produced expected results, low Reynolds numbers resulted in much larger decreases in centerline density. Additionally, the density did not increase post-constriction, rather remained relatively constant. Since low Reynolds numbers are associated with the flow in microchannels, it will be interesting to compare this result with experimental data when it becomes available. In this Thesis it was assumed that the height of the channel, $L_{z}$ was large enough to assume $z$ independence. One challenge that may arise experimentally is designing a flow domain where this assumption will still be valid.

Lastly, the role of the second-order derivative was investigated. Previous work done by ([24], [20]) discarded the second-order term in equation (3.26) and solved the first-order quadratic differential equation. With the inclusion of the second order term it was observed that pressure did not decrease as much as predicted by the first-order solution. Also, the minimum density is achieved at a position that is downstream of the maximum constriction.

In the future, MPC methods can be used to study the flow properties of the fluid using a particle based approach. The results obtained from MPC could then be compared with the results presented in this Thesis. It would also be interesting to directly compare the solutions obtained from an idealized cylindrical model with the results from the rectangular model. For the analytic approach one may ask: How is the solution affected by the axisymmetric nature of the cylindrical model? Using MPC methods one may ask: How is the solution affected by the corners that arise from the rectangular geometry? 


\section{Appendix A}

\section{Additional Calculations}

\section{A.1 Derivatives of Integrals}

Consider the following property of multi-variable functions:

$$
\frac{d}{d y} \int_{0}^{R} h(x, y) d x=h(R, y) \frac{d R}{d y}+\int_{0}^{R} \frac{\partial}{\partial y} h(x, y) d x
$$

Equation (A.1) will be used to in the derivation of the pressure gradient to move derivatives outside of integrals. For the model chosen:

$$
\begin{aligned}
\frac{d}{d y} \int_{0}^{R} v d x & =\frac{v_{s}}{\sqrt{1+R^{\prime 2}}} \frac{d R}{d y}+\int_{0}^{R} \frac{\partial v}{\partial y} d x \\
\frac{d}{d y} \int_{0}^{R} \rho v d x & =\frac{\rho v_{s}}{\sqrt{1+R^{\prime 2}}} \frac{d R}{d y}+\int_{0}^{R} \frac{\partial(\rho v)}{\partial y} d x \\
\frac{d}{d y} \int_{0}^{R} v^{2} d x & =\frac{v_{s}^{2}}{1+R^{\prime 2}} \frac{d R}{d y}+\int_{0}^{R} \frac{\partial v^{2}}{\partial y} d x \\
\frac{d^{2}}{d y^{2}} \int_{0}^{R} v d x & =\frac{v_{s}}{\sqrt{1+R^{\prime 2}}} \frac{d^{2} R}{d y^{2}}-\frac{2 v_{s}}{\left(1+R^{\prime 2}\right)^{\frac{3}{2}}}\left(\frac{d R}{d y}\right)^{2} \frac{d^{2} R}{d y^{2}}+\int_{0}^{R} \frac{\partial^{2} v}{\partial y^{2}} d x
\end{aligned}
$$


Here the boundary condition $\left.v\right|_{x=R}=\frac{v_{s}}{\sqrt{1+R^{\prime 2}}}$ from Section 3.1 has been used.

\section{A.2 Centerline Velocity}

Below are the details for the derivation of (3.14) from the flow rate analysis. Starting from equation $(3.13)$ :

$$
\begin{aligned}
\bar{V}= & \frac{1}{R} \int_{0}^{R} V\left(A \eta+B \eta^{2}+C \eta^{3}+D \eta^{4}+E\right) d x \\
= & \frac{V}{R} \int_{0}^{R}\left[A\left(\frac{R-x}{R}\right)+B\left(\frac{R-x}{R}\right)^{2}+C\left(\frac{R-x}{R}\right)^{3}+D\left(\frac{R-x}{R}\right)^{4}+E\right] d x \\
= & \frac{V}{R}\left[A\left(\frac{1}{2} R\right)+B\left(\frac{1}{3} R\right)+C\left(\frac{1}{4} R\right)+D\left(\frac{1}{3} R\right)+E(R)\right] \\
= & V\left[\frac{1}{12}(12-\lambda+T+2 M-12 E)+\frac{1}{18}(3 \lambda-3 T)+\frac{1}{24}(-12-3 \lambda+3 T-6 M+12 E)\right. \\
& \left.+\frac{1}{30}(6+\lambda-T+4 M-6 E)+E\right] \\
= & V\left[\frac{7}{10}-\frac{1}{120} \lambda+\frac{1}{120} T+\frac{1}{20} M+\frac{3}{10} E\right] \\
= & \frac{7 V}{10}-\frac{1}{120} \frac{R^{2}}{\mu} \frac{d P}{d y}+\frac{1}{120} \frac{R^{2} \rho g}{\mu}-\frac{1}{5} \frac{R^{2}}{H^{2}}\left(V-v_{s}\right)+\frac{3}{10} \frac{v_{s}}{\sqrt{1+R^{\prime 2}}}
\end{aligned}
$$

Now, expanding and solving for $V$ in terms of $\bar{V}$, leads to equation (3.14), namely:

$$
V=\frac{1}{\left(7 H^{2}-2 R^{2}\right)}\left(-\frac{1}{12} \frac{R^{2} H^{2} \rho g}{\mu}+\frac{1}{12} \frac{R^{2} H^{2}}{\mu} \frac{d P}{d y}-2 R^{2} v_{s}-3 \frac{v_{s} H^{2}}{\sqrt{1+R^{\prime 2}}}+10 H^{2} \bar{V}\right)
$$

\section{A.3 Derivative of $V$}

In Section 3.3, the derivative of the centerline velocity is needed for constructing the ODE, equation (3.26). Differentiating equation (3.14) with respect to $y$ results in: 


$$
\begin{aligned}
\frac{d V}{d y} & =\frac{1}{12\left(7 H^{2}-2 R^{2}\right)}\left[-2 \frac{R R^{\prime} \rho g H^{2}}{\mu}-\frac{R^{2} g H^{2}}{\mu} \frac{d \rho}{d y}+2 \frac{R R^{\prime} H^{2} k_{B} T}{\mu m} \frac{d \rho}{d y}+\frac{R^{2} H^{2} k_{B} T}{\mu m} \frac{d^{2} \rho}{d y^{2}}\right. \\
& \left.-60 \frac{Q H^{2}}{L_{z} \rho^{2} R} \frac{d \rho}{d y}-60 \frac{Q H^{2} R^{\prime}}{L_{z} \rho R^{2}}-48 R v_{s} R^{\prime}+36 \frac{v_{s} H^{2} R^{\prime}}{\left(1+R^{\prime 2}\right)^{\frac{3}{2}}} \frac{d^{2} R}{d y^{2}}\right] \\
& -\frac{1}{12\left(7 H^{2}-2 R^{2}\right)^{2}}\left[4 \frac{R^{3} \rho g H^{2} R^{\prime}}{\mu}-4 \frac{R^{3} k_{B} T H^{2} R^{\prime}}{\mu m} \frac{d \rho}{d y}-240 \frac{H^{2} R^{\prime} Q}{L_{z} \rho}+96 R^{3} v_{s} R^{\prime}\right. \\
& \left.+144 \frac{v_{s} H^{2} R R^{\prime}}{\sqrt{1+R^{\prime 2}}}\right]
\end{aligned}
$$

Here the definition of the flow rate, $Q=2 L_{z} \rho R \bar{V}$, and $\frac{d Q}{d y}=0$ has been used.

\section{A.4 An Expression for $\left.\left(\frac{\partial v}{\partial x}\right)\right|_{x=R}$}

The details for the calculation of $\left.\left(\frac{\partial v}{\partial x}\right)\right|_{x=R}$ that is used at the end of Section 3.3 are presented below.

$$
\begin{aligned}
\left.\left(\frac{\partial v}{\partial x}\right)\right|_{x=R} & =\left.\frac{\partial}{\partial x}\left[V\left(A \eta+B \eta^{2}+C \eta^{3}+D \eta^{4}+E\right)\right]\right|_{x=R} \\
& =\left.V\left[A\left(-\frac{1}{R}\right)+2 B \eta\left(-\frac{1}{R}\right)+3 C \eta^{2}\left(-\frac{1}{R}\right)+4 D \eta^{4}\left(-\frac{1}{R}\right)\right]\right|_{x=R} \\
& =-\frac{V A}{R} \quad\left(\text { where }\left.\eta\right|_{x=R}=0\right)
\end{aligned}
$$

Use the expression for $A$ that we developed in Section 3.1 to expand equation (A.13). This leads to:

$$
\begin{aligned}
\frac{V A}{R} & =\frac{V}{R}\left(2+\frac{1}{6} \frac{R^{2} \rho g}{\mu V}-\frac{1}{6} \frac{R^{2}}{\mu V} \frac{d P}{d y}-\frac{4}{3} \frac{R^{2}}{H^{2}} \frac{\left(V-v_{s}\right)}{V}-\frac{2 v_{s}}{V \sqrt{1+R^{\prime 2}}}\right) \\
& =\frac{2 V}{R}+\frac{1}{6} \frac{R \rho g}{\mu}-\frac{1}{6} \frac{R}{\mu} \frac{d P}{d y}-\frac{4}{3} \frac{R}{H^{2}}\left(V-v_{s}\right)-\frac{2 v_{s}}{R \sqrt{1+R^{\prime 2}}}
\end{aligned}
$$




\section{A.5 Reynolds Relationship}

An additional upstream analysis is needed to determine a relationship for the Reynolds number. Begin with equation (3.14), namely:

$$
V=\frac{1}{\left(7 H^{2}-2 R^{2}\right)}\left(-\frac{1}{12} \frac{R^{2} H^{2} \rho g}{\mu}+\frac{1}{12} \frac{R^{2} H^{2}}{\mu} \frac{d P}{d y}-2 R^{2} v_{s}-3 \frac{v_{s} H^{2}}{\sqrt{1+R^{\prime 2}}}+10 H^{2} \bar{V}\right)
$$

Now consider the upstream centerline velocity, $V_{0}$. Setting $R^{\prime}=0, H=2 R_{0}, R=R_{0}$, and $\rho=\rho_{0}$ and simplifying, leads to:

$$
V_{0}=\frac{20}{13} \overline{V_{0}}-\frac{7}{13} v_{s}+\frac{1}{78} \frac{R_{0}^{2}}{\mu}\left(\frac{d P}{d y}-\rho_{0} g\right)
$$

Now, solving the Navier-Stokes Equation $(2.1)$ with $\mathbf{u}=(u, v, 0)=(0, v(x), 0)$ we get:

$$
0=\mu v_{x x}-\frac{d P}{d y}+\rho_{0} g
$$

Integrating with respect to $x$ results in:

$$
v(x)=\frac{1}{2 \mu}\left(\frac{d P}{d y}-\rho_{0} g\right) x^{2}+C_{1} x+C_{2}
$$


where $C_{1}$ and $C_{2}$ are unknown constants. Now impose the following conditions:

$$
\begin{aligned}
\left.\left(\frac{d v}{d x}\right)\right|_{x=0} & =0 & \text { (Symmetrical velocity profile) } \\
\left.v\right|_{x= \pm R_{0}} & =v_{s} & \text { (upstream slip velocity) }
\end{aligned}
$$

Using (A.18) and (A.19) we can solve for $C_{1}$ and $C_{2}$ in (A.17) to obtain:

$$
v(x)=\frac{1}{2 \mu}\left(\frac{d P}{d y}-\rho_{0} g\right) x^{2}-\frac{R_{0}^{2}}{2 \mu}\left(\frac{d P}{d y}-\rho_{0} g\right)+v_{s}
$$

Using the definition of centerline velocity, $\left.v(x)\right|_{x=0}=V$, the upstream centerline velocity from (A.20) becomes:

$$
V_{0}=-\frac{R_{0}^{2}}{2 \mu}\left(\frac{d P}{d y}-\rho_{0} g\right)+v_{s}
$$

Substituting the new upstream relationship (A.21) into (A.15) and solving for $V_{0}$ we get the new upstream relationship:

$$
V_{0}=\frac{3}{2} \overline{V_{0}}-\frac{1}{2} v_{s}
$$

Rewriting this in terms of flow variables, and noting that $\frac{d P}{d y}=0$ upstream, equation (A.21) becomes:

$$
V_{0}=\frac{\rho_{0} g R_{0}^{2}}{2 \mu}+v_{s}
$$


Substituting (A.23) into (A.22) and solving for $\overline{V_{0}}$ gives:

$$
\overline{V_{0}}=\frac{1}{3} \frac{\rho_{0} g R_{0}^{2}}{\mu}+v_{s}
$$

In Section 2.3, the dimensionless quantity Reynolds number (3.25) was introduced. This relationship must be true upstream, which gives:

$$
R e_{0}=\frac{\rho_{0} \overline{V_{0}} R_{0}}{\mu}
$$

Substituting the expression for the upstream average velocity in (A.24) into (A.25) gives:

$$
\begin{aligned}
R e_{0} & =\frac{\rho_{0} R_{0}}{\mu}\left(\frac{1}{3} \frac{\rho_{0} g R_{0}^{2}}{\mu}+v_{s}\right) \\
& =\frac{1}{3}\left(\frac{\rho_{0} \bar{V}_{0} R_{0}}{\mu}\right)^{2} \frac{g R_{0}}{\bar{V}_{0}^{2}}+\left(\frac{\rho_{0} \bar{V}_{0} R_{0}}{\mu}\right) \frac{v_{s}}{\bar{V}_{0}} \\
& =\frac{1}{3} \operatorname{Re}_{0}^{2} \frac{g R_{0}}{\bar{V}_{0}^{2}}+\operatorname{Re}_{0} \frac{v_{s}}{\bar{V}_{0}}
\end{aligned}
$$

Now, solving for $R e_{0} \neq 0$ gives:

$$
R e_{0}=\frac{3\left(1-v_{s_{0}}\right)}{g_{0}}
$$

where $v_{s_{0}}=\frac{v_{s}}{\bar{V}_{0}}$ is the scaled, upstream slip velocity and $g_{0}=\frac{g R_{0}}{\bar{V}_{0}^{2}}$ is the scaled, upstream force term. This is the result presented in Section 2.4. 


\section{A.6 Density ODE}

In Section 3.3 the density gradient was developed using Maple. It has the form:

$$
0=\Omega_{1}\left(\frac{d^{2} \rho}{d y^{2}}\right)+\Omega_{2}\left(\frac{d \rho}{d y}\right)^{2}+\Omega_{3}\left(\frac{d \rho}{d y}\right)+\Omega_{4}
$$

where the coefficients $\Omega_{1}, \Omega_{2}, \Omega_{3}$, and $\Omega_{4}$ are non-constant coefficients dependent on $y$. The worksheet used in Maple to develop this ODE is presented. Note the following translations between the worksheet and this Thesis:

- $d r h o=\frac{d \rho}{d y}, d 2 r h o=\frac{d^{2} \rho}{d y^{2}}$

- $d R=\frac{d R}{d y}, d 2 R=\frac{d^{2} R}{d y^{2}}$

- $V_{c}=V$, and $d V_{c}=\frac{d V}{d y}$

where

$$
\begin{aligned}
& V=\frac{1}{\left(7 H^{2}-2 R^{2}\right)}\left(-\frac{1}{12} \frac{R^{2} H^{2} \rho g}{\mu}+\frac{1}{12} \frac{R^{2} H^{2}}{\mu} \frac{d P}{d y}-2 R^{2} v_{s}-3 \frac{v_{s} H^{2}}{\sqrt{1+R^{\prime 2}}}+10 H^{2} \bar{V}\right) \quad \text { and } \\
& \frac{d V}{d y}=\frac{1}{12\left(7 H^{2}-2 R^{2}\right)}\left[-2 \frac{R R^{\prime} \rho g H^{2}}{\mu}-\frac{R^{2} g H^{2}}{\mu} \frac{d \rho}{d y}+2 \frac{R R^{\prime} H^{2} k_{B} T}{\mu m} \frac{d \rho}{d y}+\frac{R^{2} H^{2} k_{B} T}{\mu m} \frac{d^{2} \rho}{d y^{2}}\right. \\
& \left.\quad-60 \frac{Q H^{2}}{L_{z} \rho^{2} R} \frac{d \rho}{d y}-60 \frac{Q H^{2} R^{\prime}}{L_{z} \rho R^{2}}-48 R v_{s} R^{\prime}+36 \frac{v_{s} H^{2} R^{\prime}}{\left(1+R^{\prime 2}\right)^{\frac{3}{2}}} \frac{d^{2} R}{d y^{2}}\right] \\
& \quad-\frac{1}{12\left(7 H^{2}-2 R^{2}\right)^{2}}\left[4 \frac{R^{3} \rho g H^{2} R^{\prime}}{\mu}-4 \frac{R^{3} k_{B} T H^{2} R^{\prime}}{\mu m} \frac{d \rho}{d y}-240 \frac{H^{2} R^{\prime} Q}{L_{z} \rho}+96 R^{3} v_{s} R^{\prime}\right. \\
& \left.\quad+144 \frac{v_{s} H^{2} R R^{\prime}}{\sqrt{1+R^{\prime 2}}}\right]
\end{aligned}
$$




$$
\begin{aligned}
& {\left[>\text { density } O D E:=\left(\frac{4}{3} \frac{R^{2}}{\rho}-\frac{11}{3024} \frac{R^{4} \rho d R^{2} T k b v s}{\mu^{2} \operatorname{Vbar} m\left(d R^{2}+1\right)^{3 / 2}}\right.\right.} \\
& -\frac{1}{9072} \frac{R^{6} \rho^{2} d R^{2} T g k b}{\mu^{3} \operatorname{Vbar} m\left(d R^{2}+1\right)}+\frac{5}{2268} \frac{R^{6} \rho \operatorname{VckbT}}{\mu^{2} \operatorname{Vbar} H^{2} m\left(d R^{2}+1\right)} \\
& -\frac{5}{2268} \frac{R^{6} \rho k b T v s}{\mu^{2} \operatorname{Vbar} H^{2} m\left(d R^{2}+1\right)}-\frac{71}{15120} \frac{R^{4} \rho d R^{2} T V c k b}{\mu^{2} \operatorname{Vbar} m\left(d R^{2}+1\right)} \\
& +\frac{5}{2268} \frac{R^{6} \rho d R^{2} T V c k b}{\mu^{2} \operatorname{Vbar} H^{2} m\left(d R^{2}+1\right)}-\frac{5}{2268} \frac{R^{6} \rho d R^{2} T k b v s}{\mu^{2}{\operatorname{Vbar~} H^{2} m\left(d R^{2}+1\right)}^{2}} \\
& -\frac{1}{9072} \frac{R^{6} \rho^{2} k b T g}{\mu^{3} \operatorname{Vbar} m\left(d R^{2}+1\right)}-\frac{71}{15120} \frac{R^{4} \rho \operatorname{VckbT}}{\mu^{2} \operatorname{Vbar} m\left(d R^{2}+1\right)} \\
& -\frac{11}{3024} \frac{R^{4} \rho k b T v s}{\mu^{2} \operatorname{Vbar} m\left(d R^{2}+1\right)^{3 / 2}}+\left(\frac{1}{9072} \frac{R^{6} \rho k b^{2} T^{2}}{\mu^{3} \operatorname{Vbar~m}^{2}\left(d R^{2}+1\right)}\right. \\
& \left.\left.+\frac{1}{9072} \frac{R^{6} \rho d R^{2} T^{2} k b^{2}}{\mu^{3} \operatorname{Vbar} m^{2}\left(d R^{2}+1\right)}\right) d r h o\right) d 2 r h o+\left(-\frac{8}{3} \frac{R^{2}}{\rho^{2}}\right. \\
& -\frac{1}{9072} \frac{R^{6} \rho d R^{2} T g k b}{\mu^{3} \operatorname{Vbar} m\left(d R^{2}+1\right)}-\frac{1}{9072} \frac{R^{6} \rho k b T g}{\mu^{3} \operatorname{Vbar} m\left(d R^{2}+1\right)} \\
& \left.+\frac{5}{18144} \frac{R^{5} \rho d R k b^{2} T^{2}}{\mu^{3} \operatorname{Vbarm}^{2}\left(d R^{2}+1\right)}+\frac{5}{18144} \frac{R^{5} \rho d R^{3} T^{2} k b^{2}}{\mu^{3} \operatorname{Vbarm}^{2}\left(d R^{2}+1\right)}\right) d r h o^{2}+( \\
& -\frac{5}{2268} \frac{R^{6} \rho d R^{2} V c g}{\mu^{2} \operatorname{Vbar} H^{2}\left(d R^{2}+1\right)}+\frac{5}{2268} \frac{R^{6} \rho d R^{2} g v s}{\mu^{2} \operatorname{Vbar}^{2}\left(d R^{2}+1\right)} \\
& -\frac{71}{15120} \frac{R^{4} \rho d V c k b T}{\mu^{2} \operatorname{Vbar} m\left(d R^{2}+1\right)}+\frac{5}{6} \frac{R^{2} k b T}{\mu \operatorname{Vbar} m}+\frac{1}{9072} \frac{R^{6} \rho^{2} g^{2}}{\mu^{3} \operatorname{Vbar}\left(d R^{2}+1\right)} \\
& -\frac{1}{3} \frac{R d R v s}{\operatorname{Vbar} \rho \sqrt{d R^{2}+1}}-\frac{25}{2268} \frac{R^{5} \rho d R^{3} T k b v s}{\mu^{2} \operatorname{Vbar} H^{2} m\left(d R^{2}+1\right)} \\
& +\frac{25}{2268} \frac{R^{5} \rho d R^{3} T V c k b}{\mu^{2} \operatorname{Vbar} H^{2} m\left(d R^{2}+1\right)}-\frac{25}{2268} \frac{R^{5} \rho d R k b T v s}{\mu^{2} \operatorname{Vbar~H}^{2} m\left(d R^{2}+1\right)} \\
& +\frac{25}{2268} \frac{R^{5} \rho \operatorname{Vc} d R k b T}{\mu^{2} \operatorname{Vbar} H^{2} m\left(d R^{2}+1\right)}+\frac{5}{2268} \frac{R^{6} \rho d R^{2} T d V c k b}{\mu^{2} \operatorname{Vbar~H}^{2} m\left(d R^{2}+1\right)} \\
& -\frac{71}{5040} \frac{R^{3} \rho V c d R k b T}{\mu^{2} \operatorname{Vbar} m\left(d R^{2}+1\right)}-\frac{5}{9072} \frac{R^{5} \rho^{2} k b T g d R}{\mu^{3} \operatorname{Vbar} m\left(d R^{2}+1\right)} \\
& -\frac{71}{5040} \frac{R^{3} \rho d R^{3} T V c k b}{\mu^{2} \operatorname{Vbar} m\left(d R^{2}+1\right)}-\frac{5}{9072} \frac{R^{5} \rho^{2} d R^{3} T g k b}{\mu^{3} \operatorname{Vbar} m\left(d R^{2}+1\right)}
\end{aligned}
$$




$$
\begin{aligned}
& +\frac{5}{2268} \frac{R^{6} \rho d V c k b T}{\mu^{2} \operatorname{Vbar} H^{2} m\left(d R^{2}+1\right)}-\frac{71}{15120} \frac{R^{4} \rho d R^{2} T d V c k b}{\mu^{2} \operatorname{Vbar} m\left(d R^{2}+1\right)} \\
& -\frac{11}{1008} \frac{R^{3} \rho d R k b T v s}{\mu^{2} \operatorname{Vbar} m\left(d R^{2}+1\right)^{3 / 2}}-\frac{11}{1008} \frac{R^{3} \rho d R^{3} T k b v s}{\mu^{2} \operatorname{Vbar} m\left(d R^{2}+1\right)^{3 / 2}} \\
& +\frac{71}{15120} \frac{R^{4} \rho d R^{2} V c g}{\mu^{2} \operatorname{Vbar}\left(d R^{2}+1\right)}-\frac{5}{2268} \frac{R^{6} \rho V c g}{\mu^{2} \operatorname{Vbar} H^{2}\left(d R^{2}+1\right)} \\
& +\frac{5}{2268} \frac{R^{6} \rho g v s}{\mu^{2} \operatorname{Vbar} H^{2}\left(d R^{2}+1\right)}+\frac{11}{3024} \frac{R^{4} \rho d R^{2} g v s}{\mu^{2} \operatorname{Vbar}\left(d R^{2}+1\right)^{3 / 2}} \\
& +\frac{1}{9072} \frac{R^{6} \rho^{2} d R^{2} g^{2}}{\mu^{3} \operatorname{Vbar}\left(d R^{2}+1\right)}+\frac{71}{15120} \frac{R^{4} \rho V c g}{\mu^{2} \operatorname{Vbar}\left(d R^{2}+1\right)} \\
& \left.+\frac{11}{3024} \frac{R^{4} \rho g v s}{\mu^{2} \operatorname{Vbar}\left(d R^{2}+1\right)^{3 / 2}}\right) d r h o+\frac{2 V c}{\operatorname{Vbar}}-\frac{2 R v s d R^{2} d 2 R}{\operatorname{Vbar}\left(d R^{2}+1\right)^{3 / 2}} \\
& -\frac{2 v s}{\operatorname{Vbar} \sqrt{d R^{2}+1}}+\frac{76}{567} \frac{R^{5} \rho V c^{2} d R}{\mu \operatorname{Vbar} H^{4}\left(d R^{2}+1\right)}+\frac{23}{126} \frac{R^{3} \rho d R v s^{2}}{\mu \operatorname{Vbar}^{2}\left(d R^{2}+1\right)^{3 / 2}} \\
& +\frac{71}{5040} \frac{R^{3} \rho^{2} d R^{3} V c g}{\mu^{2} \operatorname{Vbar}\left(d R^{2}+1\right)}+\frac{11}{1008} \frac{R^{3} \rho^{2} d R^{3} g v s}{\mu^{2} \operatorname{Vbar}\left(d R^{2}+1\right)^{3 / 2}} \\
& +\frac{76}{567} \frac{R^{5} \rho d R^{3} V c^{2}}{\mu \operatorname{Vbar} H^{4}\left(d R^{2}+1\right)}+\frac{11}{1008} \frac{R^{3} \rho^{2} d R g v s}{\mu^{2} \operatorname{Vbar}\left(d R^{2}+1\right)^{3 / 2}} \\
& -\frac{263}{630} \frac{R^{3} \rho V c^{2} d R}{\mu \operatorname{Vbar}^{2}\left(d R^{2}+1\right)}-\frac{263}{630} \frac{R^{3} \rho d R^{3} V c^{2}}{\mu \operatorname{Vbar} H^{2}\left(d R^{2}+1\right)} \\
& +\frac{37}{315} \frac{R \rho V c d R v s}{\mu \operatorname{Vbar}\left(d R^{2}+1\right)^{3 / 2}}+\frac{76}{567} \frac{R^{5} \rho d R^{3} v s^{2}}{\mu \operatorname{Vbar} H^{4}\left(d R^{2}+1\right)} \\
& +\frac{71}{5040} \frac{R^{3} \rho^{2} d R V c g}{\mu^{2} \operatorname{Vbar}\left(d R^{2}+1\right)}+\frac{37}{315} \frac{R \rho d R^{3} V c v s}{\mu \operatorname{Vbar}\left(d R^{2}+1\right)^{3 / 2}} \\
& +\frac{23}{126} \frac{R^{3} \rho d R^{3} v s^{2}}{\mu \operatorname{Vbar} H^{2}\left(d R^{2}+1\right)^{3 / 2}}+\frac{76}{567} \frac{R^{5} \rho d R v s^{2}}{\mu \operatorname{Vbar} H^{4}\left(d R^{2}+1\right)} \\
& -\frac{263}{945} \frac{R^{4} \rho V c d V c}{\mu \operatorname{Vbar} H^{2}\left(d R^{2}+1\right)}+\frac{71}{15120} \frac{R^{4} \rho^{2} d R^{2} d V c g}{\mu^{2} \operatorname{Vbar}\left(d R^{2}+1\right)} \\
& +\frac{37}{315} \frac{R^{2} \rho d R^{2} d V c v s}{\mu \operatorname{Vbar}\left(d R^{2}+1\right)^{3 / 2}}+\frac{263}{1890} \frac{R^{4} \rho v s d V c}{\mu \operatorname{Vbar} H^{2}\left(d R^{2}+1\right)} \\
& +\frac{367}{630} \frac{R^{2} \rho d R^{2} V c d V c}{\mu \operatorname{Vbar}\left(d R^{2}+1\right)}-\frac{5}{2268} \frac{R^{6} \rho^{2} d V c g}{\mu^{2} \operatorname{Vbar} H^{2}\left(d R^{2}+1\right)}
\end{aligned}
$$




$$
\begin{aligned}
& -\frac{23}{378} \frac{R^{4} \rho v s d V c}{\mu \operatorname{Vbar} H^{2}\left(d R^{2}+1\right)^{3 / 2}}-\frac{152}{2835} \frac{R^{6} \rho v s d V c}{\mu \operatorname{Vbar} H^{4}\left(d R^{2}+1\right)} \\
& +\frac{152}{2835} \frac{R^{6} \rho V c d V c}{\mu \operatorname{Vbar} H^{4}\left(d R^{2}+1\right)}-\frac{152}{567} \frac{R^{5} \rho d R^{3} V c v s}{\mu \operatorname{Vbar} H^{4}\left(d R^{2}+1\right)} \\
& -\frac{152}{2835} \frac{R^{6} \rho d R^{2} d V c v s}{\mu \operatorname{Vbar} H^{4}\left(d R^{2}+1\right)}+\frac{152}{2835} \frac{R^{6} \rho d R^{2} V c d V c}{\mu \operatorname{Vbar} H^{4}\left(d R^{2}+1\right)} \\
& -\frac{5}{2268} \frac{R^{6} \rho^{2} d R^{2} d V c g}{\mu^{2} \operatorname{Vbar} H^{2}\left(d R^{2}+1\right)}-\frac{263}{945} \frac{R^{4} \rho d R^{2} V c d V c}{\mu \operatorname{Vbar} H^{2}\left(d R^{2}+1\right)} \\
& +\frac{263}{1890} \frac{R^{4} \rho d R^{2} d V c v s}{\mu \operatorname{Vbar} H^{2}\left(d R^{2}+1\right)}-\frac{23}{378} \frac{R^{4} \rho d R^{2} d V c v s}{\mu \operatorname{Vbar}^{2}\left(d R^{2}+1\right)^{3 / 2}} \\
& +\frac{25}{2268} \frac{R^{5} \rho^{2} d R^{3} g v s}{\mu^{2} \operatorname{Vbar}^{2}\left(d R^{2}+1\right)}-\frac{25}{2268} \frac{R^{5} \rho^{2} d R^{3} V c g}{\mu^{2} \operatorname{Vbar} H^{2}\left(d R^{2}+1\right)} \\
& +\frac{25}{2268} \frac{R^{5} \rho^{2} d R g v s}{\mu^{2} \operatorname{Vbar} H^{2}\left(d R^{2}+1\right)}-\frac{25}{2268} \frac{R^{5} \rho^{2} d R V c g}{\mu^{2} \operatorname{Vbar} H^{2}\left(d R^{2}+1\right)} \\
& -\frac{23}{126} \frac{R^{3} \rho V c d R v s}{\mu \operatorname{Vbar} H^{2}\left(d R^{2}+1\right)^{3 / 2}}+\frac{263}{630} \frac{R^{3} \rho d R^{3} V c v s}{\mu \operatorname{Vbar} H^{2}\left(d R^{2}+1\right)} \\
& +\frac{263}{630} \frac{R^{3} \rho \operatorname{Vc} d R v s}{\mu \operatorname{Vbar} H^{2}\left(d R^{2}+1\right)}-\frac{23}{126} \frac{R^{3} \rho d R^{3} V c v s}{\mu \operatorname{Vbar} H^{2}\left(d R^{2}+1\right)^{3 / 2}} \\
& -\frac{152}{567} \frac{R^{5} \rho \operatorname{Vc} d R v s}{\mu \operatorname{Vbar} H^{4}\left(d R^{2}+1\right)}-\frac{4}{3} \frac{R^{2} V c}{\operatorname{Vbar} H^{2}}+\frac{4}{3} \frac{R^{2} v s}{\operatorname{Vbar} H^{2}}+\frac{R v s d 2 R}{\operatorname{Vbar} \sqrt{d R^{2}+1}} \\
& +\frac{5}{18144} \frac{R^{5} \rho^{3} d R g^{2}}{\mu^{3} \operatorname{Vbar}\left(d R^{2}+1\right)}+\frac{71}{15120} \frac{R^{4} \rho^{2} d V c g}{\mu^{2} \operatorname{Vbar}\left(d R^{2}+1\right)} \\
& +\frac{37}{315} \frac{R^{2} \rho d V c v s}{\mu \operatorname{Vbar}\left(d R^{2}+1\right)^{3 / 2}}+\frac{367}{630} \frac{R^{2} \rho \operatorname{Vc} d V c}{\mu \operatorname{Vbar}\left(d R^{2}+1\right)} \\
& +\frac{5}{18144} \frac{R^{5} \rho^{3} d R^{3} g^{2}}{\mu^{3} \operatorname{Vbar}\left(d R^{2}+1\right)}-\frac{103}{252} \frac{R \rho d R v s^{2}}{\mu \operatorname{Vbar}\left(d R^{2}+1\right)} \\
& +\frac{367}{1260} \frac{R \rho V c^{2} d R}{\mu \operatorname{Vbar}\left(d R^{2}+1\right)}+\frac{367}{1260} \frac{R \rho d R^{3} V c^{2}}{\mu \operatorname{Vbar}\left(d R^{2}+1\right)}-\frac{5}{6} \frac{R^{2} \rho g}{\mu \operatorname{Vbar}} \text { : }
\end{aligned}
$$




\section{References}

[1] T. Akhter and K. Rohlf. Quantifying compressibility and slip in multiparticle collision (MPC) flow through a local constriction. Entropy, 16:418-442, 2014.

[2] H. I. Andersson, R. Halden, and T. Glomsaker. Effects of surface irregularities on flow resistance in differently shaped arterial stenoses. Journal of Biomechanics, 33:1257-1262, 2000.

[3] S. Bedkihal, J.C. Kumaradas, and K. Rohlf. Steady flow through a constricted cylinder by multiparticle collision dynamics. Biomech Model Mechanobiol., 12:929-939, 2013.

[4] S.A. Berger and L-D. Jou. Flows in stenotic vessels. Annu. Rev. Fluid Mech., 32:347-382, 2000.

[5] S. Chakravarty and P. K. Mandal. Numerical simulation of casson fluid flow through differently shaped arterial stenoses. Math. Phys., 65:767-782, 2014.

[6] Y. I. Cho, L. H. Back, D. W. Crawford, and R. F. Cuffel. Experimental study of pulsatile and steady flow through a smooth tube and an atherosclerotic coronary artery casting of man. $J$. Biomechanics, 16:933-946, 1983.

[7] M.D. Deshpande, D.P. Giddens, and R.F. Mabon. Steady laminar flow through modelled vascular stenoses. J. Biomechan., 9:165-174, 1976. 
[8] R. Feng, M. Xenos, G. Girdhar, W. Kang, J. W. Davenport, Y. Deng, and D. Bluestein. Viscous flow simulation in a stenosis model using discrete particle dynamics: a comparison between DPD and CFD. Biomech Model Mechanabiol, 11:119-129, 2012.

[9] J. H. Forrester and D. F. Young. Flow through a converging-diverging tube and it's implications in occlusive vascular disease-I: Theoretical development. J.Biomechanics., 3:297-385, 1970.

[10] J. H. Forrester and D. F. Young. Flow through a converging-diverging tube and it's implications in occlusive vascular disease-II: Theoretical and experimental results and their implications. J.Biomechanics., 3:1-25, 1970.

[11] A. Lamura and G. Gompper. Numerical study of the flow around a cylinder using multi-particle collision dynamics. Eur. Phys. J. E, 9:477-485, 2002.

[12] J. S. Lee. Numerical studies of fluid flow through tubes with double constrictions. International Journal for Numerical Methods in Fluids., 11:1113-1126, 1990.

[13] W. Liao, T. S. Lee, and H. T. Low. Numerical studies of physiological pulsatile flow through constricted tube. Emarald., 14:297-385, 2003.

[14] J.C. Misra and G.C. Shit. Role of slip velocity in blood flow through stenosed arteries: A non-Newtonian model. J. Mech. Med. Biol., 7:337-353, 2007.

[15] P. Neofytou and D. Drikakis. Effects of blood models on flows through a stenosis. International Journal for Numerical Methods in Fluids, 43:597-635, 2003.

[16] Y. Nubar. Blood flow, slip, and viscometry. Biophys. J., 11:252-264, 1971.

[17] S. Ookawara and K. Ogawa. Flow properties of Newtonian and non-Newtonian fluid downstream of stenosis. Journal of Chemical Engineering of Japan, 33:582-590, 2000.

[18] R. L. Panton. Incompressible flow (3rd Ed). John Wiley and Sons Inc., 2005. 
[19] S. Poyiadji, K. Housiadas, K. Kaouri, and G. Georgiou. Asymptotic solutions of weakly compressible Newtonian Poiseuille flows with pressure-dependent viscosity. European Journal of Mechanics B/Fluids, 49:217-225, 2015.

[20] S. Rabba. Pressure curves for compressible flow with slip through constricted cylinders. Masters Thesis, Applied Mathematics, Ryerson University, Toronto, 2014.

[21] D. S. Sankar and U. Lee. Mathematical modeling of pulsatile flow of non-Newtonian fluid in stenosed arteries. Communications in Nonlinear Science and Numerical Simulation., 14:2971$2981,2009$.

[22] G.C. Shit and S. Majee. Pulsatile flow of blood and heat transfer with various viscosity under magnetic and vibration enviornment. Journal of Magnetism and Magnetic Material., $388: 106-115,2015$.

[23] J.R. Culham W.A. Khan and M.M. Yovanovich. Fluid flow and heat transfer in power-law fluids across circular cylinders: Analytical study. Journal of Heat Transfer., 128:870-878, 2006.

[24] L. Yao and D. Li. Pressure and pressure gradient in an axisymmetric rigid vessel with stenosis. Applied Mathematics and Mechanics (English Edition), 27:347-351, 2006. 